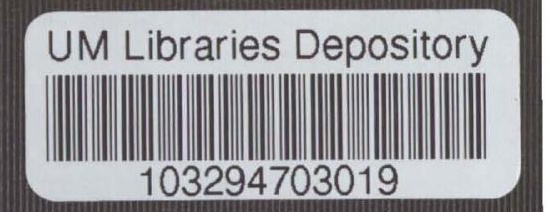



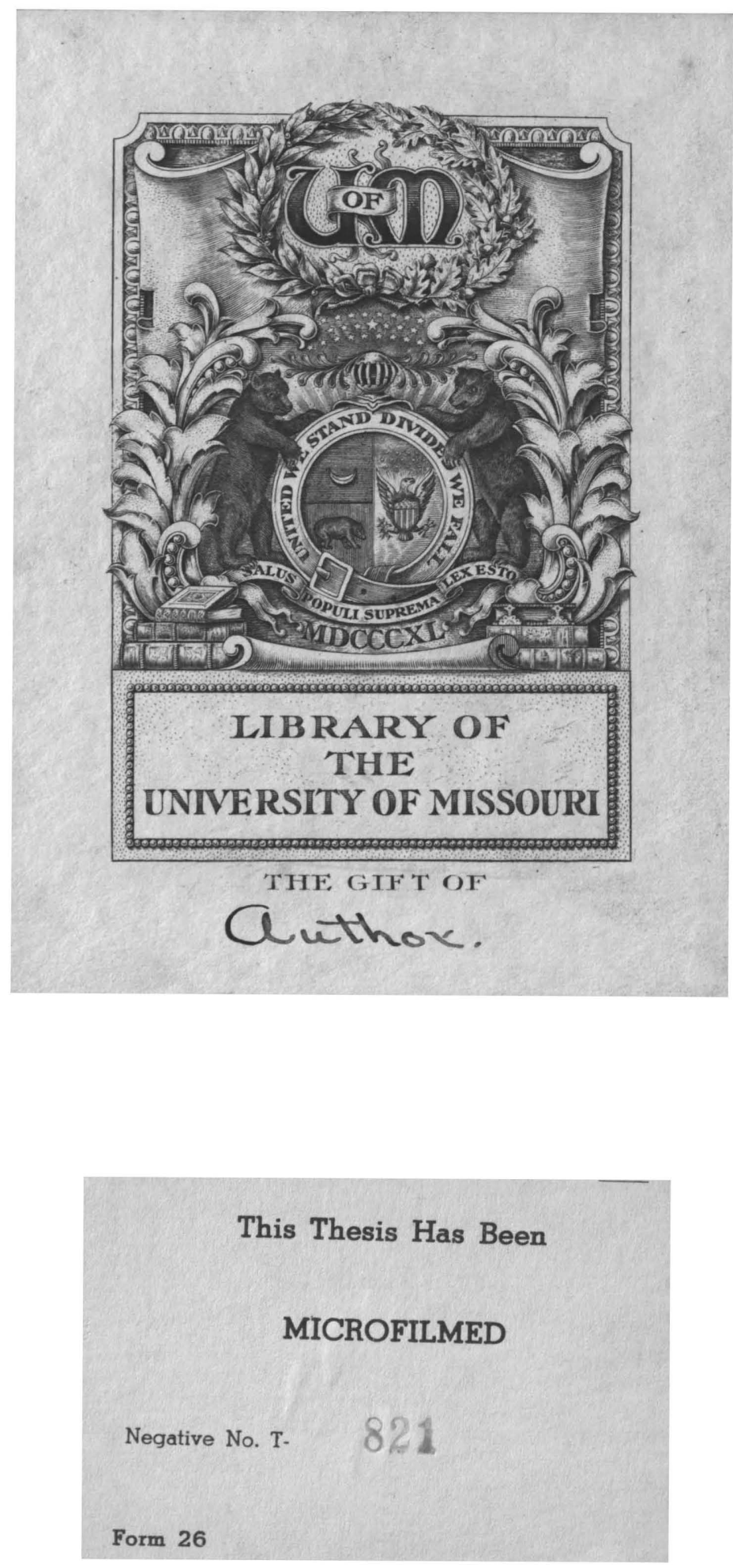






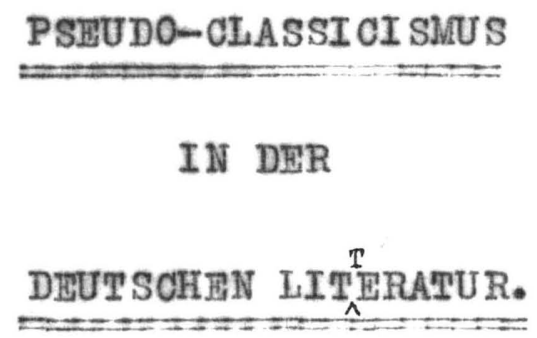

A THESIS SUBMITTED FOR THE DEGPEE

MASTER OF APTS.

GIIDA C. KIRCHNER, A. B.

MISSOURI STAPE UNIVERSTTY.

COLUMBIA, MO. 

1. Rthckblick auf den Zustand Deutschlands im sechzehnten und siebzehnten Jahrhundert.--Politische, sociale, religibse und litterarische Verhalt tnisse.

a) Das sechzehnte Jahrhundert.--Die Reformation, Sprache, Litteratur und Regierung.

b) Das siebzehnte Jahrhundert.--Der dreissigjuhrige Krieg, die Renaissance --Die Sprache, Litteratur, Regierung, das sociale Leben.

2. Pseudo-Classicismus --stufen der Entwickelung:+t

1) Einflhrung und Herrschaft des franzbsischen Einflusses.Opitz als Dictator in der deutschen Litteratur.--Fran:zBsische und hollandische Vorbilder.

2) Nachahmung italienischer Vorbilder: Marini.--Entartung in Marinismus .--Hoffmanm und Lohenstein, die hervorragendsten Dichter dieser Art.

3) Franzbsischer Classicismus wieder von Canitz eingeruhrt; erreicht seinen Hohepunkt in Gottsched.

4) Einfluss der englischen Schriftsteller: Addison, steele, Pope, Shaftesbury, Thomson.--Moralische Wochenschriften. Naturbeschreibungen von Brockes und Haller.--Bodmer und Breitinger.

5) Kampf zwischen franzbsischen und englischen Einflussen.-Gottsched auf der einen, Bodmer und Breitinger auf der anderen Seite.--Entschiedener Sieg des englischen Einflusses.

3. Herrschaft des Pseudo-Classicismus gebrochen.

1 Klopstock befreit die deutsche Litteratur von den engen Fesseln des Pseudo-Classicismus +++ Grthde flur seinen Erfolg: *

a) Zustand Deutschlands unter Fried.II von Preussen.

b) Franzbsischer Einfluss: Voltaire, Diderot, Rousseau .

c) Englischer Einfluss: Milton, Shakespeare.

d) Geistiger Zustand des Volkes: Pietismus, Aufkllorung. (Spener, Leibnitz, Wolf.)

2. Wieland, Herder, Lessing, die Vorlkufer der zweiten clas\$sischen Periode in der deutschen Iitteratur.

4. Bedeutung des Pseudo-Classicismus fur die deutsche litteratur:-Der Zusammenhang mit der folgenden classischen Periode.-Vergleichung mit den thnlichen Perioden in den Litteraturen der Franzosen, Englander, Italiener.--Der Pseudo-Classicismus bildet eine Vorbereitungsstufe zum Classicismus. 



\section{PSEUDO- CLASSICISMUS}

in der

DEUTSCHEN IIITTRATUR.

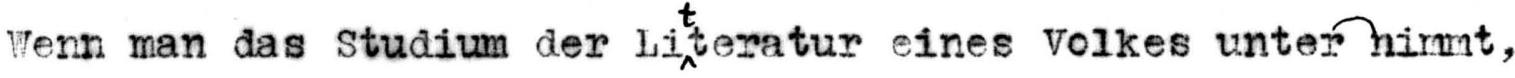
stellt sich die Thatsache klar heraus, dass die Literatur nicht von dem Leben, Charactertund Idealen des Volkes getrennt, sondern vielmehr mit desselben eng verbunden ist. Sie ist in wirklichkeit das Verzeichniss des nationalen Geistes, der Ausdruck ces moralischen ,religioesen und geistigen Zustandes des volkes.

Die Vorbedingung zur Bildung einer National-Litteratur ist eine festhnationale Gmundlage des Volkes. Es moegen vielleicht beruehmte Schriftsteller, Genien sogar , ohne diese Gmundlage entstehen,aber wenn diese Basis fehlt,wenn die nationele Entrickelung des Volkes gehindert worden ist, se kann die Litheratur desselben keine hohe stufe der vollendung erreichen.

Diesen Rusamenhang zwischen dem Leben und der Litteratur eines Volkes wird eine Betrachtung der deutschen Litteratur im sibbzehnten Jahrhundert klar beweisen. Whe jedoch eine einigermassen gruendiche Untersuchung dieser Iitterarischen Periode unternommen werden kann,wird es noetig sein,einen Rueckblick auf den zustand Deutschlands im sechzehnten und im Anfang des siebzehnten Jahrhunderts zu werfen, um besser verstehen zu koennen, was eine solche Litteratur moeglich machte.

Der zweck dieser schrift ist jedoch nicht, diese Periode kue st lerisch zu betrachten und kritisispen, sondern sie von dem culturfoge- 

schichtlichen Standpunkte aus zu bexhandeln; auf die verhaeltnisse einzugehen, aus welchen eine solche Iitteratur entstand, die Entwickelung des Pseudo-classicismus stufenweise zu betrachtenund schliesslich den Zusammenhang dieser Periode mit der folgenden classischen herauszustellen.

Eu einer Zeit, als England, Frankreich, die Niederlande und mindert. andere staaten turopas das nationale beben in sich erweckten,foerderten und entwickelten und die Grundlage zu einer National-Lit ${ }_{1}$ teratur bildeten,lag Deutschland in einem solchen politischen und socialen Zustand, dass der nationale Geist und das volkstuemliche Gefuehl fast ganz und gar erstickte, und dadurch eine weitere Intwickelung der Litteratur unmoeglich gemacht wurde.

$\frac{\text { Lussicht }}{a m}$ $\frac{\text { Anfang }}{\text { des }}$ dahrinu-

Die Aussicht, an Anfang des sechzehnten Jahrhunderts eine lit teratur auf einer nationalen Gmundage, gestalten zu koennen, var scheinbar sehr guenstig. Die humanistische Bewegung gegen mittelalterliche Ideen the Regierung und Gelehrsamkeit, der Binfluss solcher Maemer wie Hutten, Frasmus und Luther, oeffneten den Weg zu einer neuen, hoeheren Form des nationalen Lebend.Geistig war Deutschland bereit, diesen Weg zu betreten. Iuther befreite das Gomissen, er berrite die Deutschen vor Joch der Tradition; er schuf aus den vielen verschiedenen deutschen lundarten durch die Uebersetzung der Bibel eine geltende deutsche Sprache.Der Gebrauch der deutschen sprache ist dadurch wieder in die Litinteratur eingefuehrt worden, aber das Lateinisehe war dennoch vorwiegend die Dichtersprache.

Literatur. Die Iitteratur bot manches dar, das von echtem, volkstuemlichem Geist durchdrungen war. Die religioesen Lieder waren von Tiefe der mmpindung und Innigkeit des volkstuemlichen Gefuehls durch- 

dmungen. Die İterarischen Leistungen von Hans Sachs, irther und ahderen schriftsteliern dieser Periode beriesen aie Existenz von Keimen wahrer Volkstuemlichkeit.Zu dieser Zeit war Deutschland auf dem 1itterarischen Gebiete auch nicht so weit hinter England zumeck,aber um ciese volkstuemilchen Keime zu jener Kunsthoehe zu entwickeln, zu welcher es England gebracht, forderte eine originalitaet und schoepferische Kraft, die Deutschland nicht besass. Eine Zeit lang lebte der volkstuemliche Geist noch in den Kirchenliedem, Volkstiedern und Sagen von Faust und dem evigen. Juden;aber am Ende des sechzehnten Jahrhunderts sind diese fast ganz ausgestorben, und alles ward dem steifen Gelehrtentume unerthaenig.

$\frac{\text { Gruende }}{\text { des }}$

Bueckschlages.

fimation.

Die Gruende des verderblichen Rueckschlages, wie Hettner ${ }^{*}$ sie bezeichnet, sind areifache:

1). Die ausschliesslich theologische Natur der Reformation.

2). Der zornmutige Starrsinn Luthers.

3). Der politische Zustand.

Die Reformation bot eine herrliche Gelegenheit zur vollstaendigen Fntwickelung nationaler Finhei tund geistiger und religioeser Freiheit,aber anstatt sich zur Blvete zu entfalten,erstarrte sie in Formen.In der Religion fand ein bestaendiger Kampf statt, nicht nure zischen Protestanten und Katholiken, sondern auch zwischen Lutheranem und Reformipten innerhalb der protestantischen Kírche selbst.

Politischer iustind.

Der politische, sociale und geistige Zustand bot jeinen trau. rigen Anblick dar. Deutschland war in viele kleine staaten get feit, ueber welche der Kaiser nur eine nominelle Macht hatte. Die Regiemung Karls des Fuenften hatte inren Schwerpunkt in spanien und. Itajien, und dieser trat daher mit seiner undeutschen, rein per* Siteraturgeschichte des achegehnten gahehunderto" III. I., S. 2 . 

soenlichen Politik der deutschen Reformation und dem begeisternden Nationalismus feindlich entgegen.

Socialer

und

geistiger Zustand.

Die Fuersten waren feig, habsuechtig und treulos und dachten nicht an das Wohl des volkes, sondern nur an ihren eigenen Gewinn. Die Gelehrsamkeit und die geistige Bildung stand unter dem EinPlusse der Geistlichkeit und der Fuersten. Das Volk nahm keinen Anteil an 11terarischer Ausbildung. Die Wissenschaft war durch die theologische Beschraenktheit ganz vernichtet.Selbst Luther sah in dem Altertumsstudium nur ein Mittel der Bibelerklaerung. Ex erkannte die Philosophie nur an,insqweit sie Beweis und stuetze des Glaubens war. Waehrend die Philosophet in sigland die Gmundlagen freier Forschung errichteten, diente die Philosophie in Deutschland als eine Anleitung zu eitlen theologischen streitigkeiten,welche als das ziel des ganzen geistigen Lebens gatten. Am Fnde des Jahrhunderts war Deutschland die Beute und der Hohn

Das der Fremden. ehrite Jahrhundert. reissigaehrige Krieg.

Politischer ustand am SchIusse des Kieges.

Aus dem religioesen Streit des sechzehnten Jahrhunderts entstand im siebzehnten Jahrhundert der dreissigjaehrige krieg,der Deutschland bis auf den Tod erschoepfte, und von dessen schreck1ichen Folgen es sich nie voellig erholt hat.

Der Krieg vernichtete die Sicherhejt des deutschen Kunstgeistes von Grund aus, und jede Hoffnung religioeser una politischer Freiheit war deln deutschen Volke voellig entnommen.Nationale rraditionen und Ideale waren ausgestorben.Ueberall exist. ten Roheit, Aberglaube und Rechtlosigkeit.

Am Schlusse des Krieges war das Kaiserhaus gedemuetigot und deutsche Reichseinheit voellig zertmemmert,aber aus den Truemmern ist ein neues, ganz verschiedenes Scheinreich entstanden. 

Die Fuspten und Staende stehen nicht mehr in Rejchsrecht sondern im Voelkerrecht. Territorien waren zu Staaten, die Freiheit der Fuersten zur hoechston ewalt, das Reich zum roelkerrechtichen Bund geworden. Deutschiand war in etwa zweihundert unabhaengige Fuerstentuemer eingeteilt, und das Princip "cujus regio,ejus religio"war alleinherrschend. Mit dem schwindenden Machtbewusstsein schwand auch der letzte Hauch von Vaterlandsliebe und volkstuemlichem Gefuehl. Deutschland noerte auf in wahrheit deutsch zu sein - Die Deutschen sahen in Ludwig dem vierzennten einen zweiten Karl den Grassen, der adzu bestimmt sei, Deutschland von deil drohenden politischen Gefahren zu retten."Ich bin Kajser in meinem Lande "ist das Motto der Fuersten in Deutschland am Schlusse des Krieges und ueberall entstanderkleine fuerstilched Hoefe nach dem Muster Ludwig des Vierzehnten gebildet. Der ganze politische Zustand deutet auf die innerliche Pagulniss aen Reiches hin.

ReIiioese und OCiaIe Verhaeltnisse.

Was die Folgen des Krieges auf den religioesen und socialen Zustand waren, kann man sich kaum vorstellen. In ganz Deutschland befand sich am Ende des Krieges kaum eine Milizon Binwohner;BerIin hatte etwa dreihundert Buerger.Das Leben war fuer das Volk im Allgeneinen nur ein Kampl um ein blosses, freudenloses Dasein. Die meisten deutschen Fuersten kannten kein hoeheres ziel als die komoediantenhafte Nachaeffung der franzoesischen Practliebe, Verschwendung und Liederlichkeit.Ihr ganzes Leben war schamlos. Aber neben dem fuerstlichen Absolutismus herrschte auch der kirchliche. Durch den westfalischen Frieden waren beide Religionen, die protestantische und die katholische anerkannt,aber der roligioese streit war dennoch nicht zu Bnde gebracht.Der ReIfgion, wie dem ganzen socialen Leben, fehlte es an Tiefe der Fm- 

prinding, was aus den Fnigrammen und Satiras cer Zeit hervorgent Eins yon Logaus epigramen lavtet, zum Beispiel, wie folgt "Luthrisch, Paopsischland calvinisch, diese glauben aIze cre

Sind rorhanden, doch 1st zweifel, wo das Christenthum dann sei." und w1eder:

"Dieweil Peligion bestent in cemuete

"Tie, dase man sie dann sucht mit Iisen im Gebluete"

Logau beklagt sich auch ueber die "Alamote-Kleider"und das"Alamode - Xinnen" " Wie sich's randelt aussen, wandelt sich's auch inneny

Gleicher Art sind mehrere anderen satirischen 3ilde? lieser periode.

Die: interatur und das geistige Leben.

Das geistige Leben Deutschlands am Anfanc des siebzennten Jahrhunderts war fast verstockt. Die Fuersten, Geistlichen und Gelehrten varon die machtvollsten staonde, und we Gelehrsamkeit und geistige Bildung des volkes stand unter inren Tinfusse. Jep Buegger war kleinstaedtisch und ohne allen inneren schmung. Tast siebzig Procent der gesammten Bevoelkemung war nit iston ueberbuerdet und fuenrte ein elendes, meonticohes vrc carun verstocktes Leben.

\section{Verdortung der}

In den Kreisen aer Gelerrten unc dor Geistichreit fand die geutscher lateinische sprache Past ausschliessliche Pflege. Je mehr die spracie.

Reformation yon ihrer urgpmenglichon polkstuemlichen pichtunc abrich, umpdmehr gewann das Lateinicche die Gewalt ueber das Deutsche. Die lateinische Sprache nurde ir laus und schule gelehrt,und sie hatte die deutsche als die lichterorrache ersetzt. Fuer das wissenschafticiche Denken ging die deutsche sprache fast voellig verlonen.M1t einem deutschen Buche konnte sich der ver- 

Iasser keinen him erwerben. Nur unter den volke fand sie noch Pflege, denn sie war in ihrer damaligen Form fuer das gemeine Leben ausgebildet und wurde auch deshalb von den fuersilichen und gelehrten Staenden verachtet.

An Hofe wurde die cleutsohe sprache durch de armutige, fliessende frangoesische ersetzt. Eine gurendilche Kenntniss und der Gebrauch der Pranzoesischen Sprache war das Kennzeichen eines fein gebilasten llannes. Durch den Tinfluss des Adels, den Lom Volke das Verlangen erregt hatte, auch fein und gebildet "nv sein, murden Pranzoesische woerter in die deutsche Sprache eingefuehrt und mit den deutschen woertern vermengt, so dasidie reine deutsche sprach ganz verloren ging. Nurch die Finfuehmug des Lateinischen als (die) Schriftsprache, war das volk von der Iittematur und der geistigen Bildung fast gaenzlich ausgeschrossen.

An den hoeheren schuien und Untversitaeten mrde lateinisch, oenr renig Gulechisch, Mathematik und Theologie gelehrt.Das neue Testament wirde ausschiesslich oareson. nio Taunsache man die Glaubenslehre - Der Theologte fohlte aber eine wissenscharticie Gmundage, undaie echte missenschaft, das Ideal der Humanisten, 18t bis auf den Namen verschwinden.

Anregung su nouer Thaotigkeit.

Dieses stockende Leben, welches au dieser reit in nevtschIanc bostand, musto man wiedor in Bowogung setzen. Deutschrand. musste ron inem nationalen,patrtotischen feist ergrifeen und zu nouor thatigkelt angespornt werden, um es aus einem sclchen zustande wu retten. Derjentge,derfaies an Inde volibrachte, frar Friderich der Grosse. Irotz seiner Verkenmung der deutschen Iit tomatur war er der Befreier der Deutschen. Aber onnekevisce rorherettendo Anfaenge haette er dieses herrliche work nicht vollbringen koennen.

Die Stufen der Intwickelung des geistigen Zustandes des 

Volkes vor Priedrich dem Zweiten, wie Hettner sie andeutet, sind:

1). Die Renaissance.

2). Die Lebenstrage der Hissenschaft und die Abwerfung des theologischen Joches.

3). Der Pietismus des edlen Spener.

4). Der Rationalismus, durch welchen der Weg der Wissenschaft witer, abert sicherer wurde.

Die letzten arei fallen inrephaze de letztef Haelfte des siebzehnten Jahmundents und. werden spaeter erklaert werden.Man Ienke die Aufmerksamkeit daher auf die erste Stufe, die Renaissance, welche sich am Anfang des siebzehnten Jahrhunderts von ItaIien ${ }_{n}$ uns

Nach den schon emraehnteh politischen und socialon Zustaenden Deutschlanda zu dieser Zeit,kann man leicht verstehen, dass die Renaissance in Deutschland aine ganz andere Richtung einschlagen musste, wie in den anderen Isendern Buropas. Fngland, Fronkreich, die Niederlande, selbst Italien, Spanien und PortugaJ. standen an Fnde des sechzehnten Jahmhunderts auf esstem, nationalem Bodenfund verarbeiteten nach kurger geit das Fremde vollstaendig,und erobern auf der Grundlage der Renaissance eine neve eigenartigo,volkstuomiliche Kunst und Dichtung,

durch shakeso net ist.

Deutschland dagegen, in seiner kirchlichen und staatilchen Verkommenheit, war ohne eine solche nationale cmundrage. Jeder zwingende innerliche Trieb und alle schoepferische Kraft fehlte dem Deutschen zu dieser Zeit. Sie vereieren mehr und mehr der aeu-sserlichsten Nachahnung und verloren zuletzt alle visserschaftIiche und kuenstlerische Selbstaendigkeit und utspruenglichkeit. * Siteratur geschichte des achtzehnten gahihunderts. III. 1., S. 24. 

Die Rentisible ty Joutsonland durchraeuft jene mannigfastaissance $\rightarrow$

ne Auferweckung der nationalen Vergangenheit, wie es zum Beisrie: in Italien der Fall var, Die deutsche Renaissance ist asus-encicher und geistzoser, wie irgend wo ancers, wath ihr keine selbstaendige und heimischt schoeperische kraft entgegen komnt.

Die Renaissance machte sich zuerst in suedrevtschland fuehlbar. sie lehrte verschi-dons vel kanschauungen und erveckte wieder die Liebe zum studium des Altertums una nes Antike。 Die litterarischen Geister zu der Zeit sahen in der Nachahmung der, classiker und deren Nacholmes wie einzige Rettung der deutschen lit teratur. Aber Deutschland konnte den echten poetischen $=i d$ dor - Jassischen Dichtkunst nicht erfassen und daher entfitand eine pseudo-classische Dic tung, dio sich skIaviswis an die classischen Formen hielt, aber den wirklichen poetischen tahalt der ganz verfehlte.

In anderon iaendern wurde neben der lateinischen Dichtung $\mathbf{s}$

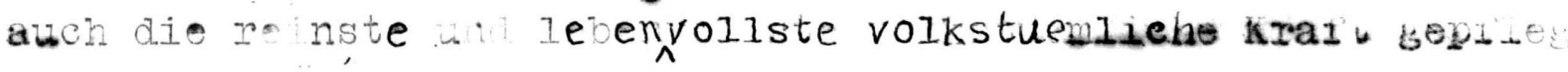
In Deutschland tritt die Renaissance fast ausschliessijch in der toten,einseitig-gelehrten Form der neulateinischen Dichtung auf und kennt keinen anderen Kuassitab als den der Nuetzlichkeit. cismus. Dieser Pseudo-classicismus in der deutschen Literatur, der mit der Renajssanoe anfeengt und sich durch das ganze Jahrhunder hindurchzleht,ist vielmehr eine Nachahmung der erfolgreichen Nachahmer der classischen Dichtung, naemlich der Franzosen und Italiener,als der Classiker selbst; das heisst,es war keine directe Nachahmung der classiker, und daher der Name pseudo oder falscher classiciomus.

Ws fehlte disser pseudo-classischen I $\frac{f_{\lambda}}{\lambda}$ eratur an einem un- 

mḯtelharen Zusammenhang mit den Gmunsaetzen des wirklichen Lebens und an einem nationalen Geiste. Strenge Beobachtung gewisser steifer Regein war der Grundzug dieser Dichtung , was die Folge hatte, ine blosse, hohle, kalte Formvollendung, ohne die vesent11che, begelsternde, poetische kraft hervorzubringen.

D1e Aufgabe waere nun jetzt, dom Intwickelungsgang dieser pseudo-elassischon Litteratur zu folgen und der bestaendigen EvoIution des volkes zur joechsten Bildung nachzugehen, aus welcher politische und religiosse Frothelt und die swelte classische $\mathrm{Pe}-$ , riode in der Iitteratur hervorgingen.

Stufen der

Dor Pseudo-Classiciemus in Deutschland sehlug in wirklichintWickelung keit mur dre1 verschiedene Richtungen ein,aber waohrend der Herrdes PseudoC19881cismus. Echaft der lotzton Richtung ontstanden zwol andere Stroemungen, welche einen dauernden und gesunden Pinfluss auf die Litteratur. ausuebten. Man koennte daher die Entwickelung dieser littemaischen periode durch fuenf stufon bezeichnen -

STTJTN DER RNTWIOKELUNG IM PBHUDOMUAASICISMUS.

C:27:2:

1. Finfuehrung und Herrschaft des franzoesischen Einflusses. opitz als Dictator in der Littoratur. italienischer

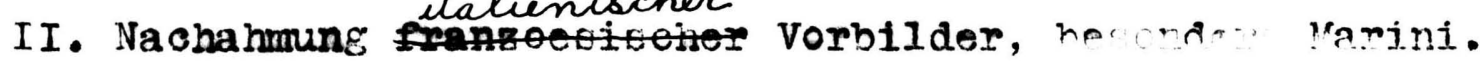
Fntartung in hartnismus - Hothann une Icrerstein, tie inervorragendston Dichter dieser Art.

III. Franzoesischer Classicismus wieder alJe1nherrschend; ongofuhrt von

eingefuehrt 7 on canitz, orreicht selnen yoehepunkt in Gottsched.

IV. Einfluss der englidohen Schriftsteller, Addison, steel, Pope u.a.- Brocker, Haller, Bodner und Breitinger.

v. Kampe zwischen franzoestschen und onglischen ginfluessen, Gotisched auf der einen, Bodmer und Brej- 

tinger auf der anderen Seite. Entschiedener Sieg des englischen tinflusses, Herrschaft des Pseudo-classicismus gebrochen.

Finen weitlaeufigen Bericht ueber die Leistungen eines je-

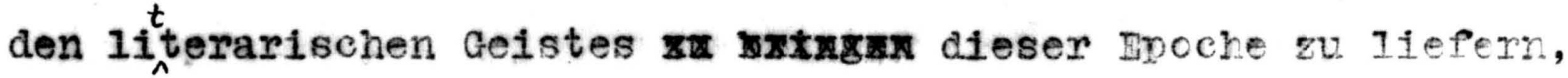
ist nicht der Zweck dieser Schrift. Wir verden uns daher nur an die Haupttalente halten, die den groessien Einfluss auf die Literatur ausuebten und die Dichter von geringerem wert mur in ueb-g-han erwaehnen。

\section{EINTURHRUNG UND HERRSCHAFT DES FRANZOESISCHEN EIN-}

\section{FLUSSES.}

Das centrum der Thaetigkeit dieser Periode bilden die Iit terarischen Leistungen opitzens. Es wird jedoch vorteilhaft sein, die folgenden Abteilungen in Betracht zu ziehen:

a). Vorlarufor Opitzens.

a). Vorlaeufer opitzens und Sprachgeselischaften.

b). Opitz und die erste schlesische Dichterschule.

c). Volkstuemilcher Hauch in der Dichtung. Kirchenlieder, Dichtungen von Fleming, Dach und anderen.

a). VorIaeufer pitzens. sich ueber Deutschland verbreitete und zu neuer linterarischer
Als zu Anfang des siebzehnten Jahrhunderts die Renaissance Thaetigkeit anspornte, regte sich das Gefuehl unter den meisten gebildeten Maennem, auch fuer Deutschland eine National-Literatur wieder zu schaffen. Der erste Schritt jedoch zur wiederherstellung der Literatur war die Reinigung der deutschen sprache von Frendwoertorn und inre wiedereinfuehrung in die titieratur. Die Dichtersprache des sechzohnten Jahehunderts war Lateinisch, die des siebzehnten sollte Beutsch sein.

Die ersten, welche die Notwendigkeit der Pelege der Mutter- 

sprache anerkanntenund in reges Interesse fuer dioselbe erveckten , waren Danaisius, Melissus, Zinkgref und Weckherlin.

Veckher-

1in.

SprachEesel1Echafton
Der letzt erwaehnte war eigentlich der Vorlaeufer und Zeitgenesse von opitz, und an dichteriso hem Talent war or inm wit ueclen. In Geist, Leidenschaft und charakter bildete er die Frgaenzung zu. Opitz. $\mathbb{F r}$ besass ein grosses talent fuer Sprachen, fuehrte die suodliohon pormen und Versmasse, die sonnette, Sestinen, Alezandriner, Eclogen und oden in die deutsche Litheratur ein, und pelegte seine Gedichte in der deutschen sprache angufertigen. Wr war aber kein Dichter son Professionfund deutete nur den Weg an, welohen opitz spaeter betrat und sich dadurch dauernden furm erwarb. Dem Regelzwang der Opitz'schen Dichtung konnte Weckherlin sich nicht unterverfen, und in seinen Gedichten erlavbl er sich oft gross Freiheit im Versbau.

Die Anregung zur Wiederpflege der deutschen sprache und die Begeistorung, ,olch die schon erwaehnten Maenner dafuer zoigtent fuehrte zur Begmiendung verschiedener Goselischaften, deren zvock os war, die Muttersprach zu roinigen und zu versuchen, sie wiedor in inrer voelligen wherde herzustelien. Das studium der Antike wurde auch das Ziel dieser Gesellschaften. Diese Sprachfesellschaften, wie man sie nannio, wurden nach dem torbilditalienischer Akademien gestiftet, und inre Mitgljeder hestanden fast ausschliessIich aus Maennern adeliger Herkunft.

Dieses war in einer Hinsicht oin gutes Zeichen, dern es zeigte, dass der Hof, dass die Tonangeber anfingen sich von dem franzoesischen Joch losgusagenserst nach otwa einhundert Jahren fand

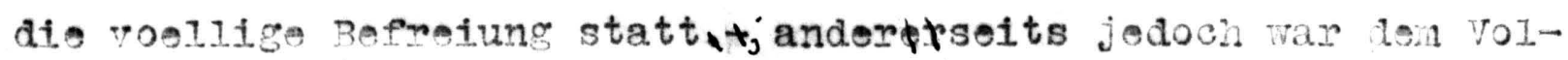
ke Zutritt in diese Gesellschaften verschlossen. Die 1itterari- 

des siobvehnton Jahrhundarts, wie ite des zwoelf e.,

was daher mit den Interessen aes Adels eng verbunden;aber die Aristokratie des zwoelften Jahrhunderts wurde heren Idealen boseistert, und daher war auch ihre Litteratur weit bedeutender und von echtem, Iitterarischem Geiste beseelt.

Die hervorragendsten Sprachvereine waren: "Der Palmenorden",oder die "fruchtbringende Gesellschaft", die"deutschgesinnte Genossentschaft", die "Gesellschaft der Pegnitzschaefer", oder der "gekroente Blumenorden". Sprachgesellschaften zu stiften, wurde in Deutschland, wie wte os zu der Zeit auch in Italien und Frankreich der Fall war, eine foerriliche Mode. Sie hatten alle denselben Zveck, Prlege des classischen Studiumslund Saeuberung den deutschen sprache.

Die Beschreibung einer dieser Geselischaften wird genuegen,

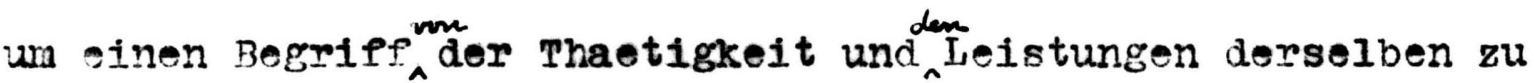
liefern.Man betrachte daher die aelteste und bedeutendste, naemlich"die fmachtbringende Gesellschaft", die ix 1617, gerade ein Jahr vor dem Ausbruche des Krieges, von Ludwig, Fuerst von Anhalt,

Gostiftet und bis zum Jahre 1680 bestand.

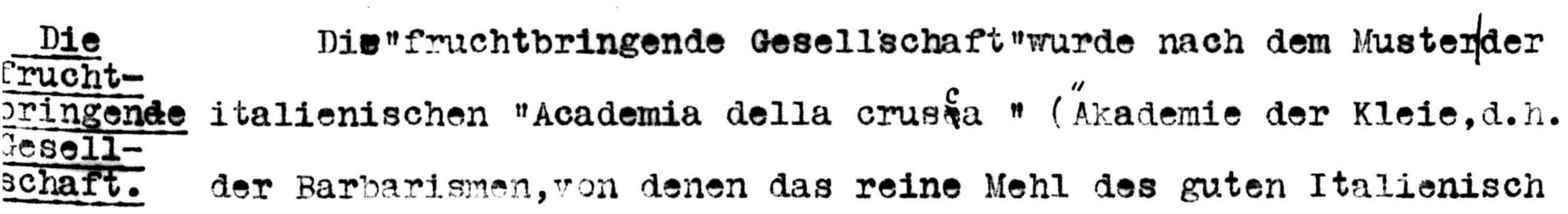
Sese11schart. der Barbarimen, ron denen das reine Mehl dos guten Italienisch gesaeubert varden solite)gebildet. Der Plan der Finrichtung war ? gang naci im itatipnischen vorbilde gefasst. Als Symbol nahm die Gesellschaft den indianischen Palmbaum(Cocosmussbaum)mit dem Sinnspmach:" A1los zura Nutzon": iodes Mitglied hatte eine Pflanze, oins Blumo, odor oine Frucht, Eun Abzeicher und anon poGishsin, dem orden entsprechenden Geserlschafts-nansn. * Fuerst Iudwig hiess girn Boigniel "dor Naehrenae" und hatte -

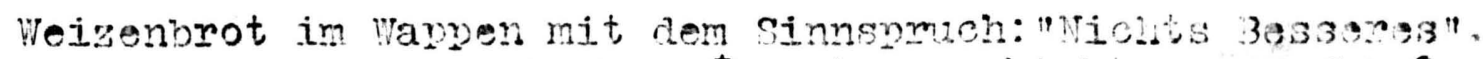



Bin anderer waehlte sich die Maiblume und vorde der wonlniechende genannt,u.s.w. Dios fuchrie natuerlioh zu laechorlichen Spielereien. Die Gesellschaft bestand aus etwa vierhundert Mitgliedern, unter denen sich neben hohen Herren und throrsts auch folehrte und Dichter bofandon, wis gus Boispial paitz, Mrophits und

\section{Uebertrej-der Gramnatiker schottel.}

bungen
infinger
sohaftent

In den selben stil waren die anderen vereine gefasst,aber in ihrem Bifer, die deutsche sprache zu reinigen,gingen einige derselben $\mathrm{gu}$ weit und verfielen in laecherliche Uebertreibung. Die deutschgesinnte Genossenschaft empfahl folgende woerter: Tagelouchter vanstatt Fenster, Schauburg anstatt Theater, Loeschnom horn oder Gesichtswerer anstatt Nase. Der "gekroente Blumenorden schlug vor,in der Poesie woerter sinnreich zu geschreiben. Der Wind sollte zum Beispiel in dieser Dichtersprache Wolkentreiber heissen; der Fruehling, Blurenvator; Blut, nasses Lebonsgold u.s. Aus diesem geht hervor, dass diose Gesellschafton in der Lit

schaften. dmok, an gmondlichon Verstaendniss der Sache. Allgomeine Sprachund Lit it turinteressen waren in Aussicht genommen, aber die Hauptbeschaertigung der Leiter wurde Nanengebung, Devisesuchung, Herrichtung von Modalitien und Tapponnalereion.Ihr Verdienst un dio Literatur bestand nicht in den Iittorarischen Productoin der Mitglieder, gondorn in der Anrogung untor den hoeneren klagsen zur Wiederpelege der deutschen Sprache and des classischen Studiuns. Sio vorkoorporn in sich das Gefushl, dass otras un die Litoratur gothan warden unss, un inr Lobon zu retton, und durch inre Bamsmungen trurde das Intorosso fuer di Mutionspracho und dio Dichtkunst wieder ervockt. 

Viel bedeutenderen und dausnden ginfluss als diese Geselischafton ausuoben konnton, hatte in schriftsteller, Martin opitz, dessen Regeln fuer die deutsche Dichtkunst massgebend fuer das ganze Jahrhundert waren.

Opitz war koin echtes dichterisches ralent,aber wonl ein golehrter und gebildetor Mam. In sueddeutschland, in schlesien, geboren,wo der Geist der Renaissanco an staerkston herrschte, rurde unser junger Dichter von siner Liebo gurn classischon studiun argriffon, und or sioht in dom studium des Altortuns und in der Nachahmung derclassiker und Renais sane-Dichter anderer Laender don wog,der deutschon poosio wioder aufunelfon. Fr worlte Puor Doutschland das schaffon, was Trissino und Rucollai fuer Italion, was spaoter Malherbe,Marot und Ronsard Puer Frankreich, und Daniel Heinsius und vondel rugr di viodertande omungen hatten. Die Premdhoit und Ausschtiosslichreit dap neulatoinischen Dionbung sollt duch oine in dor volkssprache werpasste nichtung orsetzt worden.

Opitz Opitz erkannte die Notwendigkeit an, oinen Leitraden ruer Dietator in dor itteratur. Reform mit seinem"Aristarchus", eine lateinische Rede ueber die dis loutsche Sprache und Dichtkunst zupchaffer. Ir begint seine Verachtung der deutschen Sprache, in welchem die Grundsaetze der sprachgeseilschatton hervorgehoben sind. Soine oigentliche Autoritaet jedoch als Reformator und ntetator in dor deutschen Literatur datirt rom Jahre 1624,als or sein"Buch von der deutccher Poetrey" herausgab.wit diesem Buche grif? on mit soinan Roporm durch,wurde als vater der deutschen Dichtkunst gepriesen, und galt nicht nur in boutschland, wosiers auch in anderen Laendern Buropas als der bedeutendste deutsche Dichter. 

ron $\frac{\text { Buch }}{\text { der }}$

Mit diesem Buch beginnt man die peit der neueren deutschen

ueber die Dichtkunst. Das Werk ist nicht originell.0pitz nahr viel aus Ronsard und scaliger; oertors findet man eine woertilche Intlehnung. Seine Abhandlung vober "die Errindung der Dinge" ist zum Beispiel aus Ronsards Abschnitt, Deptinvention, uebersetzt.

"Die Rrfindung dor Ringe",schreibt opitz, "ist nichts anderes, als sine sinnreiche fassung aller sachenydie wir uns einbilden koennen, die Himmlischen und jrdischen, die Leben haben und nicht haben, wolch oin poete jhm zuschreiben und herfuertue bringen vornimpt". * und die stelle ueber Disposition, "An dieser Erfindung honget stracks die abtheilung, welche besteht in inor fueglichen und artigen orcrurg cer exfurcer sacher"**

opitzens vorbilder waren Henteius, Ronsard, Scaliger und die griechischen und lateinischen dassiker - Er haelt sich aber an sirengsten an die Franzosen.

Opitz geht in diesem Buechlein auch auf die Arten der Dichtung ein, auf das Epos, die Tragoedie, die komoedie u.a. Hier pelegt er auch scaligm absuschreibenfenn or bohauptet: "Iie Tragoedie ist an dor lajestaot dom horoischen Gedichte gemaess, ohne dass sie selten leidet, dass man geringen standes personen und schechte sachen infuehre, whil sis nur von koeniglichem willen, rodtschlaegen, Verzweiplungen, indor-und vatemorden, Brande, Biusischan

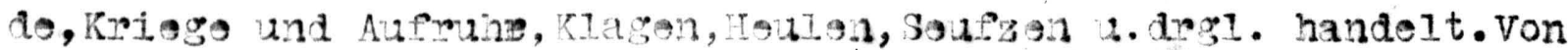
derer Zugehoer schreibt vornehmich Aristoteles und etwas weitlaeufiger Daniel Heinsius, die man lesen kann "** 

In dem letzten Satz erkennt man also seine vorbilder in dieser Gattung der Dichtkunst.

Seine Definition der Komoedie ist ebenfalls nur eine Entlehnung aus Scaligers Werk. "Die Komoedie besteht ",nach opitz, "In schlechtem wesen und personen; redet von hochzeiten, castgeboten, spielen, betmig und schalkheit der knechte, muhmraetigen Landtsknechten, buhlersachen, leichtfertigkeit der jugend, gebtze des alters, kupplerey und solchen sachen, die taeglich unter gomeinen leuten vorlauffen", "Das Drama wird in der Poeterey mit geringer Aufmerksamkeit behandelt, denn Opitz war kein Dramatiker. Eine naehere Erklaerung desselbetwar an dieser stelle erwaehnenswert, denn abe ausschliessliche Autoritaet opitzens und die in seinern Buechlein enthaltenen Regeln ueber das Drama sind. einigermassen verantwortlich fuer die Form, welche das Drama in Lohenstein, Hoffmann und anderen annahm.

In dem Abschnitt ueber Zubereitung und Zier der Worte, tritt Opitz fuer die Reinheit der Worte, Vermeidung der Fremdwoerter, aber fuer neue wortbildung ein. " Das Ansehn und die Dignitaet der poetischen Recie anlangend, bestehet dieselbe in den tropis und schematibus, wenn wir naemlich ein wort von seiner eigenthuemlichen Bedeutung aufeine andere ziehen"*** Fuer den richtigen Gebrauch der poetischen Rede weist er auf die Lateiner und Besonders auf Bcaliger hin.

Fines besonderel verdienst opitzens um die Literatur ist jedoch, dass or ein fuer alle Mal das rythmisehe Princip der deutschen Dichtkunst feststolite, und den Unterschied zwischen dem deutschen und lateinischen versbau deutlich herausstellte. -

Hier hatte opitz wider keine wirklich neue poetische Form * Borinski, Seite 82. "Buch vonder deutschen Poeterey" ** I emcke, Sabte 197. 

erfunden, sondern nur die alte, frushere, nach Tonhebung ordnende in's Leben gerufon, die im Verlauf der zeit,besonders im sechzehnten Jahrhundert,in silbenzaehlende,poetische Form ausartete. Seine Ansich ueber den Versbau ist folgende: "Nachmals ist auch ein jeder Vers entweder oin Jambicus oder Trochaicus, nicht swar, dass wir auf Art der Griechon und Lateiner eine gewis Groesse der Silben koennen in Acht nemmen, sondermalass wir aus den Accentertund den Ton exkennen, welche silbe hoch und welche niedrig gesetst soll Ferden. Wie wohl nun meines Wissens noch niemand, ich auch vor der Zeit selber nicht, dieses geneu in Acht genomen, scheint es doch so hoch vonthoethen zu sein, als hoch vonnoethen 1st,dass die Lateiner nach den quantitatibus oder Groessen der Silben ihre Verse richten und reguliren. Denn es gar einen ueblen Klang hat:

"Vonus die hat Juno nicht vermocht zu obsiegen". * Diese neue Theerie, dass der Accent und der Ton, nicht die Groesse der silbe das Metrum bestirmt,brachte Ordnung und syotem in den deutschen Versbau und beseitigte alle Unsicherheit. opitz behandelt dann die Art der Verse. Den Dactylus verbannte or ganz und gar aus der deutschen Poesie. Den Hexemeter hielt or im Deutsohen nicht ruv moeglich - Er zieht den Alerandriner vor, das herolsche Versmaas der Franzosen. Fr hielt den Alexandriner fuer geelgneter als deutschen orzaehlenden Vers, of man in der leutschen Spracho niont so kurz sein kann wie in der Iranzoesischen.

Die Alezandriner, nach opitzens Regeln gedichtet,wurden in Deutschland steif und starr.opitz dachte nicht man, den Unterschied, der fuer das ohr in elnem rranzoesischen Alexandriner mit seiner scharfon, gleichmaessig teilenden caesur und freier * Lemcke., Seite 198. "Buch, der deutschen Poeterey". 

Betonung und, einers opitz'schen gedichteten Alexandriner besteht. Auf diesen Punkt lenkte spaeter Breitinger die Aufmerksamkeit in seinem streit mit eltsched.

Am Schlusse seines Buechleins sagt opitz, dass in den kurzen, trocken dargelegten Regeln die Poesie selbst nicht zu finden sei. Naturbegabung mache den Dichter.

wiewohl auch opitz diese Thatsache anerkannte, blieb imm donnoch die Form eines Gedichtes das Hauptaugenmerk. Fr dachte njoht nur an ein gutes, sondern an ein schoenes Gedicht. Der Lehrsatz von Horaz a aut prodesse volunt, aut delectare poetae" wurde auch fuer Opitz der geltende zreck der Poesie, doch nebenbei war or von der Bedeutung der Form erfuellt. Ronsard war dazegen vom Leben und Denken des Altertums beseelt. Fr laesst sich mehr auf Untersuchungen veber charakter und Temperament der Poeten ein. Die franzoesische Litteratur ist daher keine blosse Nacharmung der Classischen Form.

Opitzens Stellung in $\operatorname{der}$ Literatur.
Die Reform der deutschen Metrik hat opitz die stellung im Fortschritt unserer Litteratur gegeben. Durch seine verbindung mit dem Adel,denn Opitz wie seine Zeitgenossen schrieben fuer. den Hof, verhalf er dem Dichterstand zu einer hoeheren Stufe. Und wenn er sich auch gar nicht mit dem wirklichen Wesen der Poesie beschaeftigte, wenn or die Form ueber den Inhalt schaetzte,wenn er ueber franzoesische Gegenstaende und nach franzoesischen Regeln auf deutsch schrieb und sprach, und die ganze Litteratur daher unter das franzoesische Joch warf, so nimmt or dennoch eine besondere, unverkennbare stellung in der Literatur Deutdchlands eih. Durch seine Aufmunterung zur Pflege der deutschen sprache, durch sein Strebeh, Interesse fuer das studium der Classiler zu erwecken, durch seine Binruehrung fast aller 

neuen Bichtarten und roeffnun ganz unhekanter etete in Jyrik und Lehredicht-, er fuelrte die oper in Deutschand oin, er $\nabla 10 \mathrm{~s}$ fie kein anderer auf deuta he poseie hin-,und endich durch seine Festsetzung des rhytmischen Princips,brachte equne ordnung und winheit in die literatur, die aescerst noetse rar, um Deutschland in den Stand zu setzen, wettere Fortschritte zu machen.

Ersto

schlesische

Opitz fand viele Anhaenger, die spater die orste schlosiDichter-sche schule gruendeten an deren Haupt opitz selbst stand. Das schule. Ziel dieser Schule war, Gedichte nach don Opitz'schen Regeln zu vorfassen. Inr dichterisches Princip rar, das die poesie belehren ergoetzen und bezobon, in klarer sprache sefasst, ind nach stre:gen Regeln gedichtet werden soll. Schrung der Pranta te und Tiefe der mpindung waren gar nicht in Betracht cenomen. Diesss Uobercewioht dor Torm ueber den Inhelt wurde bald Ianseilig und eintoenis, und fuohrte zur Reaction cegen lis steifen, strengen Regein und die Nuetzlichkeitsiehre der Schule. So entstand die zweite schiesische Dichterschule.

Phe wir jedoch nachentaf diese sohule eincehen, mit woloho die zweite stufe der Fntwickelung besint, pollen wi? noch qio gchon erraente dritte Apteliung der ersten stufe in Petracht ziehen, namlich den volkstuemlichen rauch in ler poesie.

3). VO1kgWie in der theolooischen Reform die grosse geistige BoroHauch. gung zuletzt in Fomen erstarrte,so rurce in der literarischen Reforn, die nxw guerst unter Jeckherin eine frefe rom annahm, das Vorbild opitzens und seiner steifen Regeln allmachtic. Aber neben diser sclavischen Nachahmung und Betonung der rorm des Gedichtes, lobte dennoch, wenn auch nicht sehr kraeftize oin volkstuemlicher Hauch, der zuweilen Ausdrack fand in den roici- 

oesen Liedern und in den Dichtungen solcher Dalente, vie Paul Fleming, simon Dach und Logau. Dieser volkstuemilche strom stirbt nicht aus, sonderm entwickelt sich langsam,aber bestaendig, wird

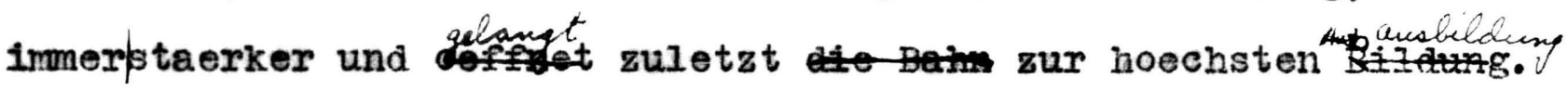
Keine Art der Dichtung hatte einen solchen gesundebtinfluss auf das volkstuemliche Leben ausgeuebt, und das maennliche Gefuehl so gluecklich erweckt, wie das religioese Lied. Der Grundzug der geistichen Lieder des siebzennten Jahrhunderts ist ein subjectiver. Die्र Lieder enthalten keinen Zug des Kampfes ẑwischen Protestanten und Katholiken, wie die des secheehnten, sondern bezeichnen das innere Verlangen einer jeden seele nach Frieden, den festen Glauben und die innige Zuversicht in Gott, der sie allein aus der draengenden Not retten kann.

Auch in der Gattung der weltlichen Lyrik besitzen wir aus dieser Zeit einige wirkliche Perlen von Paul Fleming und Simon Dach. Innigkeit des Gefuehls, Reinheit, Natuerlichkeit nebst einer gewiss f roemigkeit sind die charakterzuege ihrer Dichtungen. Frscheinungen dieser Art sind Ausnahmen in der Litteratur dieser Periode. Sie beweisen aber doch, dass der volkstuemliche Hauch noch am Leben war.

Man muss hier nicht vergessen, einen Dichter zu ermaehnen, der an poetischer vielseltigkeit und kuenstlerischer Bildung einem Opitz und Fleming nachsteht, der aber durch witz, Laune und treffenden spott, durch Vaterlandsliebe und gluehenden Fifer fuer Recht und Wahrheit alle uebertraf. Dieser Dichter var Frieda rich von Logau. Aus Logaus Bpigmmen lernen wir die oeffentlichen und privaten verhaelnisse seiner zeit kennen. Br ist der groesste Fpigrammatiker des siebzehnten Jahrhunderts, und es wird vielleicht hier am Platze sein,elnige seiner Eplgrame zu ci- 

tirinen.

1). Ueber Frankreich:

"Frankreich hat os weit gebracht, Frankreich kann

es schaffen,

Dass so manches Land und Volk wird zu seinon Affen."

2). Ueber einen Hormann:

" Bei Hofe wird kein Greis,

Wer nicht zu heucheln weiss. "

3). Tober die bluehende deutsche sprache:

- Doutsche sind so alte Leute,

Lernen doch erst reden heute.

Wenn sie lernen doch auch moliten,

Wie recht deutsch sie handeIn solitenl"

4). Ueber franzoesische Kleidung:

- Diener tragen insgenein threr Herren Lotvereá;

S011's denn sein, dass Frankreich Herr, Deutschland aber Diener sei?

Freies Deutschland, schaem'dich doch dieser schnoeden Knechtereil"

Diese werden genuegen, einen Begrif? zu geben ron den Zustaenden Deutsohlands, wie Logau sie sah, verspottete und bekaempfte, und wie or das Volk zur Besserung aufmunterte.protz all seinek Widerstandesgegen Fremdlaenderei erkannte er, ebenso wie Fleming und Dach, Op1tz als den groes ton deutschen Dichter anund nahm sich inn zum Muster.Diesepeig' wieder die ungemeine Autoritaet, die Opitz auf sein Zeitalter ausuebte.

Die erste, wennauch nicht die entschiedenste, opposition gogen die nuechterne Nuetzlichkeitslehre der ersten schlesischen Dichterschule ging von Nuernberg aus - Dort waren die Pegnitz- 

II. DER ITALIFNI SCHE EINFLUSS.

Diese stufe laesst sich in zwei Haupt+abteilungen trennen:

a). Die tinfuehrung der schaefertbichtung und des Marinismus.

b). Die zwelte Schlesische Dichter-Schule.Hauptgeister Hofrmann, Lohenstein, Gryphius.

Die erste, wem auch nicht die entschiedenste, opposition gegen die nuechterne Nuetzlichkeits-Lehre der ersten schlesischen Dichterschule ging von Nuernberg aus. Dort war der orden der Pegnitz-schaefer oder der 'gekroente Blumen-Orden gestiftet, und. die Mitglieder desselben, nach Waeme und Tiefe der mmpindung strebend, nahmen italienische vorbilder sum Muster, vor allen Marino und Guarini, und ruehrten das schaeferwesen, die spielereien der italienischen schaeferpoesien, in das deutsche Leben und Dichten ein. 

schaefer oder der gekroente Blumenorden gestiptet und die Mit-

Glieder desselben, nach Waeme und piefe der mpeindung infer

Poesie strebend, namen italienische vorbilder zum liuster, vor

allen Marino und Cuarini, und fuehrten das schaeferwesen, die spie-

lereten der italienischen Sehaeferpoesien indas deutsche Leben und Dichten ein.

Schaefer-

Die Renaissance hatte die wiederbelebung der Eclogen Vir gils und ovids mit eich gebracht. Ueber ganz Europa verbreitete sich dann des eferleben. Der Einfluss der schaeferei kam auch von England nach Deutschland mit den englischen Komoedianten, und Sidneys Arcadia fand in Deutschland grossen Beitall.

In Italien war das Schaeferwesen, das durch Sehnsucht nach natuerlichen Zustaenden sich erzeugt hatte, als Nebensache beirieben. In Deutschland ward es zum Inbegriff alier echten Poesie erhoben. Die unnatur kam in die Natur hinein; in ihrem streben nach gierlichkeit und sinnreicher sprache,gerieten die Dichter in Unnatuerlichkeit und verloren allen Halt an Einfachheit . Dieses Schaeferwesen in der Dichtkunst atteto spaeter in. Marinismus aus, was nur eine acute tirscheinung der Schaeferei tar. Marino, dessen Dichtung selbst in Abgeschacktheit verfalIen war, wurde zum Hauptvorbilde genomen. Schoehheit wird der Gegenstand der Rede im Marinismus.

Die hervormgendsten Mitglieder des Blumenordens waren Harstiarsdoerfer, Birken und Klai,und aus den Ueberschriften ihrer literarischen Producte geht der Charakter derselben hervor. Farsdoerfer zum Beispiel, erlangte Beruehmtheit durch seine "Frauenzimmergespraechespiele", eine Art Damen-Conversations-Lexicon, und durch seinen "poetischedprichter" oder"Anweisung,in 6 stunden die deutsche Reim- und Dichtkunst einzugiessen "oft auch kurz der "nuemberger Trichter"genannt.Der "Poetische Trichter" ist na- 

tuerlich gegen Opitzens langweiliges "Buch von der deutschen Pooterey" gemuenzt.

Vordienst der

PognitzSchaefer Dichter.

Die Pegnitz dichter waren der Meinunglass die Poesie vor allem in einer Stil bestehe, und sie versuchten dieses in Theorie und Praxis zu beweisen. Daraus entstand natuerlich ein unertraeglicher Bilderschrulst und suesse Trendelei. Wenn jedoch die Bemuehungen dieser Gesellschaft in plumpe, uebertriebene Nachahmung der Italiener ausartete, so verdient sie dennoch unsere Aufmerksamkeit,indem sie Betonung auf das Phantasieleben und auf die Rmpfindsarkeit legte, die der Opitzschen Poetik entschieden fehlte.

Weit entschiedener als die Nuernberger wich von opitz eine Gmppe von Dichtern, ${ }_{2}$ welche die zweite schlesische schule bildete. Diese schule hatte ihren Sitz auch in schlesien und wird daher die zweite schlesische Schule genannt.

Zweite Schrosische

Die Mitglieder dieser schule nahmen auch die neueren italiDichter-enischen Dichter zum vorbilde. Das dichterische Princip dieser schule. Schule unterscheidet sich von der ersten, indem die Poesie nur ergoetzen und nicht notwendig belehren soll. Das hatte zur Folge,ein streben nach Lieblichkeit des Ausdrucks, nach galantem stil und sinnreicher sprache, was spaeter in Uebertriebenheit verfief, und in eine suess1iche Bmpfindsamkeit und schruelstige,prunkhafte, bombastische sprache ausartete. Diese Dichter fuehrten neben dem aesthetischen einen moralischen Verfall in die Dichtkunst ein.

Die Hauptvertreter der Dichtkunst dieser Schule sind Caspar von Lohenstein und Hoffmann von Hoffmannswaldau,

$<\quad$ All die Grundzuege der Poetik der Schuls sind in den Dichtungen dieser Maenner yorhanden, und oft findet man sie in accen- 

tuijífter Form. Es waere daher hier noetig, naeher auf inre Leistungen einzugehen. lan koennte jedoch hinzufuegen, dass es fast allen Dichtern dieser schule an Feinhelt, Tiefe, Leben und wahrem dichterischem relente fohlt, und dass die charakterguege ihrer Dichtung Bombast und Unvermunft, Geschmacklosigkeit und Mischung des Fdlen und Unedlen sind. Fier duerfen wir jedoch wieder de Dichter nicht zydohare critisfien, denn der Finfluss der zeit verlangte das Oratorische und den Redepomp, und in der moralischen Verderbtheit der zeit liegt der crund der Unsittichkeit in der Dichtung.

Snamen zudieser zeit aber, wie zur zeit der ersten stufe,entskandent

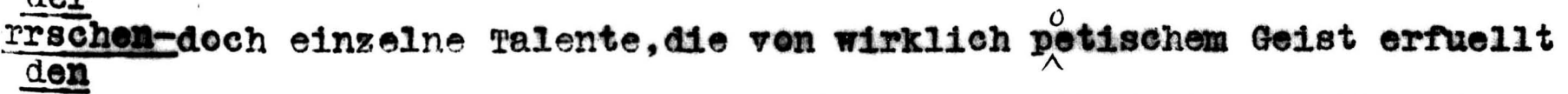
chtungs-waren. Friedrich von spee und Paul Gerhardt in der Lyrik und Anart. dreas Gryphius, besonders auf dem Gebiete des Dramas, haben Grosses in der Literatur geleistet.

Paulus Gerhardt steht naechst Luther als der bedeutendste dea Kirchonliedáxplifiter da. Er bildet den Uebergang von der kirehlichen Objectivitaet in sechzehnten Jahrhundert zur subjectivitaet des persoenlichen Gefuehlalebens. Neben einem festen Glauben und einem unerschuetterlichen Gottvertrauen geht eine hohe Freudigkelt durch Gerhardts Lieder.

Andrea, Gryphius bewies ein echt dramatisches Talent, aber durch bittere Lebenserfahrung und durch den schruelstigen FinPluss der zolt kam es nicht zur volien. Bluethe. Fr bildet den Uebergang von der orsten zur zweiten Dichterschule, und durch inn machte das Drama einen Fotschritt. Fr zelgte elne Neigung zum Shakespeare'schen und war unter den ersten, das historische schauspiel herporzuhoben, das Eomische in dem rolkstuemilohen, und das Mrnste in den eschichtilichen su pflegen. Das Dramadieser Peri- 

ode stand meistens unter dem Finflussefon Opitzens Defination desselben, aber in den Haenden von Lohenstein und anderen verfaelit diese Dichtungsart in schilderungen von schandthaten, Wut, Rache, Reue, Verzweiflung und Greuel aller Art.

Fine Aenderung in der Art der Dichtkunst maste frueher oder spaeter eintreten. Die zwelte schlesische schule fuehrte das dichterische Prinzip der Pegnitzodichter in's Bxtreme und rief dadurch oine Gegenwirkung herbei, die verfaliene Dichtkunst wieder auf gesunden, festen Boder|zu setzen. Diese Reaction und deren Din-fluss auf die Iiteratur bildet den Gegenstand der aritten stufe In der Fntwickelung dieser Periode.

\section{HERRSCHAFT DFS FRANZOESISCREN CIASSICISMUS.}

Die zweite schlesische schule fand ihren ersten Gegner in Christian Weise, der besonders auf dem Gebiete des Dramas die Poesie von dem Bombast der Unnatuerlichkeit zu befeien suchte. Obgleich eine von Grund aus unpoetische Natur, sind seine Bestrebungen fuer sittlichere und naturlichere Gestaltung der deutschen Dichtungsart jedoch unverkennbar.

Das streben nach naturwahrer Einfachhert ist gleichfalls das Ziel des Freiherrm von canitz. Canitz (+ 1699) fuehrt wieder den franzeesischen classieismus in die deutsche Litteratur ein, welcher in Gottsched seinen Hoehepunkt erreicht. Fr strebte, dureh selne satirischen und hoefischen Dichtungen die Klarheit und sleganz nachzuarmen, durch welche sich die franzlesischen Dichter auszeichneten, und der schwulst der Darstellung eine feine, natuerIiche Sprache entgegen zu setzen. Fr narm daher Boileau zum Murter, und er 1st, wie Gerrinus es ausdrueckt, "ein schwaches Echo von Boileau. "Fr besass aber kein wirklich poetisches Talent. Fs 

fehlte ilm an Lebendigkeit des Gefuehls und an hoeherem Schwung. Hofpoesie. Die Hofpoesie, wie sie von canitzens Zeitgenossen, Besser und Koenig, betrieben wurde, bestand aus Gelegenheitsgedichten und schlechten Reimereien. Der einzige, der im wahren Sinne verdient, ein Dichter genannt zu werden,war Christian Guenther. Aber wie Geothe-pen-ilm-eagt Wyr wusste sich nicht zu zaehmen und so zerrann $1 \mathrm{~mm}$ sein Leben wie sein Djehtet"* Seine Gedichte bewiesen. demnoch die Moeglichkeit einer Dichtung, die ganz aus dem Ieben zum Herzen sprach.

Der Uebergang zum franzoesischen Geschmack, den Canitz vollzog, war kein blosses zurueckgreifen auf die vorbilder opitzens, die in Frankreich selbst von der neuen Bchule des grand siecle de Louis XIV. zurueckgedraengt waren. Das Vorbild, welches canitz zum Muster narm, war Boileau, der in der"Art potique" die Gesetze der classischen ftanzoesischen Literatur verkuendete, die auch bald fuer die gesammte europaeische Kunstaichtung geltend wurân.

Derjenige, welcher am entschtedensten fuer den franzoesischen Geschmack eintrat, und der am einflussieichsten in der 1iterarischen Reform wirkte, war Johann chriatoph Gottsched. Seine Leistungen und steliung in der Iitheratur werden spaeter ausfuehriich in Betracht genormen werden. Man lenke die Aufmerksamkeit daher jetzt auf den englischen sinflus, der keine stufe fuer sich allein bildet,aber der den franzoesischen classicismus unterbrach und so maechtig ward, dass er mit dem franzoesischenloeschmack in confliot geriet, und siegreich aus dem Kenpf dahinzog. Was fuehrte nun zub binfuehrung des englischen Geschmacks waehrend der. Alleinherrschaft des franzoesischen classicismus? Den Grund muss man wieder in dem geistigen und socialen Zustand * "Dichtiong und trahrhiert. Atempel. Bd21, s. 49. 

des Volkes am Anfang des achtzennten Jahrhunderts suchen.

\section{ENGISISCTRM EINFLUSS.}

Um einen guten Begriff von dieser Periode zu bekomm, waere es vorteilhaft, sie durch die folgenden Einteilungen zu bezeichnen.

a). Geistiger und socialer Zustand Deutschlands im achtzemten Jahrhundert.

b). Einfluss Englands. Entstehung moralischer "ocherschriften.Brockes. Ha11er. Hagedorn.

Die Literatur des siebzehnten Jahrhunderts und des ersten Viertels des achtzehnten stand gar nicht mit dem Volkstuemiicher: in Beruehrung. Die Dichter warenty on keiner hohen Idee ergripeen, der sie Ausdruck geben wollten; und sie begnuegten sich daner nit Zusammensetzunger von lauttoenenden worten. Finzelne Schriftsteller erschienen aber in diesem Jahrhundert, die es sich zur Aufgabe machteb, Deutschlands herrliche vergangenheit wieder herzustellen. Diese Maenner Faren von echtem patriotischem Geiste erfrellt, und stellten sich den existipenden politischen,religioegen und socialen Zustaenden entgegen. Sie waren besonders, Iom gau, der die Religion, den Hof, den Soldatenstand und die Nachahmung der Franzosen angrifr;Moscherosch, der als der groesste Batiriker zu betrachten ist; und Grimelshausen, der bedeutendste Romanschreiber des Jahrhunderts, der in seinem "Simplieius Simplicissimus" die Eitelkeit der socialen Welt darlegt.

Je naeher man sich dem achtzehnten Jahrhundert naehert, desto entschiedener zeigt sich der abnehmende Einfluss oeffentIicher Verhaeltuisse. Dern oeffentlichen Leben lag die moralische Wuerde nicht mehr zu Grunde. Das Individuum fing nun an, sich mit 

moralischen Problemen zu beschaeftigen. Die Selbsterziehung des einzelnen Menschen wurde dem Volk als ziel vorgehalten, und indem die Litteratur teil an dieser neuen Bewegung narm,indem sie ihre Aufmerksamkeit auf die kntwickelung des inneren Lebens lenkte, und sich an den Glauben hielt, dass das innere, geistige Leou

ben gepflegt werden kann, der aasserlichen Umstaende ungeachtet, bahnte sie den Weg zur hoechsten, vollendeten Bildung.

Das achtzennte Jahr mindert. ners Ansicht die bemuste wiederaufnamme und Fortbildung der Reformations $\rightarrow+1$ deen des sechzehnten Jahrhunderts. Wissenschaftich ist es das Zeitalter der Befreiung vom Buchstaben. KuenstImisch Ist es das streben, eine eigene, selbststaendige Kunst und Litheratur zuschaffen und die Frobemung eines 1dealen, aber doch volkstuemlichen stils zu bewirken.

Erzeugnisse Thaetigkeitzu erholen. Das volk interessipt sich jetzt fuer aie Iiteratut und zeigt im allgeneinen ein verlangen nach moralischer und geistiger Bilaung. Die Kluft zwisehen gelehrter Kunstdichtung und volks11teratur faengt an zu verschwinden. Die gebildete Iitteratur wird volkstuemlicher und aie volkstuemliche gebildeter.

Die zwei Stroermugen,welche die Aufgabe der religioesen Reform wieder aufnanmen und auf intellectueletepreiheit deuteten, sind die schon erwaehnten vorbereitenden Anfaenge ror Friedrich dem Zweiten, naemilich der Pietismus und die Aufklaemung. Diese zwei Bowegungen waren bestinmt, die geistige Wiedererbauung Deutschlands zu voliziehen.

Pietjgeus. Der Pietismus ging von Spenłer und Francke aus. Sie naehprton steh und stelgerten einen widerwillen gegen ale herrschende hoelzerne Dogmatik und riefen zur tiefen Innerlichkeit. Inr stre- 

ben war nicht darauf gerichtet, das Volk im allgemeinen au begeistern, sondern das Individuum zu retten. Die Botschaft, welche die Pietisten den Deutschen brachten, war Liebe und Gottseligkeit. Aber nachdem die Bewegung aeusserlichen Frfolg erreicht hatte, verfiel aie auch in eine neue Art ${ }_{\Lambda}^{\text {or }}$ orthodox(ische) conventionalismus und entartete in Empfindelei und lystik. Aber ohne den pinfluss des Pietismus auf das deutsche volk haette die AufkIaeAuf- mang nicht inr ziel erreicht.

Die Aufklaerung war ein Teil der groesseren Bewegung, die in England durch Bacon, in Frankreich durch Descartes eingefuerrt worden war. Sie bestand wesentlich aus einer critischen Betracrtung der inneren und aeusseren WeIt vor dem hoechsten Richterstuhl, dem der Vernunft. Sie versuchte, auf systematischen wegen und mit der Hilfe der Naturwissenschaften das zu bewirken,was de Humanisten auf volksmaessige Art und Weise und vom Iituerarischen standpunkte aus unternomen hatten,

Der Weg der Wissenschaften wird weiter aber gradieniger und sicherer. Hugo Grotius, Descartes, Spinoza, BoyIe, Bacon unr I,ocke gewinnen zahlreiche deutsche Anhaenger und hinterlassen nachhaltige Spuren. In Deutschland entstehen die Philosophen, Pufendorf,Thomasius, Leibnitz und Wolf,welche die Befreiung der Wissenschaft zu stande bringen.

Aus der Aurklaerung entspross der optimistische Idealismus eines Leibnita, welche Denkart das ganze Jahrhundert herrschte. Leibnitz war der erste, der eine philosophische Denkungsart bei. der individuelien Betrachtung der Welt anbrachte. Zur Zeit der volkstuemlichen Verderbtheit bot seine Philosophie ein Obdach fuer das hoehere Leben, und naehrte die Hoffnung einer wiederm emWeckung des geistigen Deutschlands • 

Wolf folgte auf Leibnitz und indern er seine Gedanken Deutsch anstatt Iateinisch ausdrueckte, machte or zum Gemeingut, was bisher nur in den Kreisen der Gelehrten und Cobildeten war. Deutsonland Fird nun in philosophiseher und religioeser Freiheit allen Laendern Furopas ein leuchtondes Bild.

Eine solche Thaetigkeit im geistigen Leben maste auch in der Litteratur eine Veraenderung horbeifuehren. Deutachland faengt an, fuer sich zu denken, und wird daher mehr selbststaendig. Obgleich der Iranzoesische Gescmack die Literatur beherrschte, fanden die Deutbehen doeh grossen Gefalien an Addison, steele, und anderen engliøohen schriftstellern des achtzehnten Jahrhunderts. Durch die Naturschilderungen in Thomsons"Seasons", durch die Lehreedichte Popes und die Philosophie eines Shaftesbury Iernten sie der deutschon Poesie wieder einen Inhalt zu geben.

Der englische " Spectator" rief die moralischen wochenschrifschriften. ten ins Leben, die mehr als zwei Menschenalter beherrscht haben. Die deutschen Wochenschriften koen len nicht mit den englischen verglidhen werden, dem das deutsche Leben ist ganz von dem en ${ }^{\prime i-}$ schen verschieden. Der Zweck der moralischen wochenschriften war zu unterhalten und zu belehren. Die Gegenstaende, die sie behandeln, sind: Frziemug, Spiel, Geiz, Putzsucht, Verschwendung, Adelsstolz, Heirat und The, Sprachengerei u.s. . Abhandlungen ueber politische Ereignisse kommen darin gar nicht vor, denn Deutschland hatte zu der Zeit keine wirkliche Politik.

Die Behandlung der stoffe ist platt, spiessbuergerlich und beschraenkt. Nichts als breites, trockenes Moralisientren. Fs findet sich nirgends darin eine gute anmutige charakterzeichnung, wie man sie in dem englischen "Spectator" yeblickt. Die Wochenschriften verfalien endilch in handwerksmaessiget Betriebsamkeit. 

Sie waren aber doch nicht ohne Verdienst, denn sie fuehrten in Wahrhet die Literatur wieder ins Leben ein, und warfen ideale Fragen in die bildungsbeluerftige und bildungsverlassene Masse. Der englische tinfluss beschraenkte sich aber nicht aufdie volkstuemliche Richtung der moralischen wochenschriften, sondern er zeigte sich auch in den Dichtungen ton Brockes und Drollinger, die zusammen mit Hagedorn und Haller die neue deutsche nichtkunst oinleiteten. Ihre Dichtungen bestehen hauptsaechlich aus Naturbeschreibungen und Lehrgedichten und zeigen denfebergang von Boileau zu Popo. Freilich stand die englische Literatur auch seit der Stu ? und anderer franzoesischer schriftsteller, und kuenstlerisch war wenig Gewinn bei diesem Wechsel;aber die Poesie von Pope enthielt eine tiefere philosophische Weltanschauung, denn er stand unter dem Rinflusse Newtons, Lockes und des englisogen Deismus. Spaeter mit der Herausgabe von Thomsons/seasons, in welchen der Verfasser sich wieder an die Natur wendet,fand auch eine veraenderung in der englischen Litteratur statt. Deutschland, von Begeistemung fuer die Leibnitz-Holf'sche Philosophie erfuellt, fand ganz natuerlich grossen Geschmack an den \#nglaendern. Sie ahnten jedoch vielmehr deren Sohwaechen, das trockene Verstandeswesen und die absichtliche Lehrhaftigkeit, nach,als deren yorzuege.

Brockes und Haller fuehren eine neue Fpoche in die Litteratur ein. Die abgeschmack te Gelegenheitstreimereifler schlesischen Schulen und die Leere der hoefischen Dichtung wird durch sie verdraengt, und eine Dichtung von innerem Kern und Gedankenhalt, von Wuerde und Hoheit, nirmt deren Platz, die nicht nur zu bloss muessigem Frgoetzen, sondern zu einem Teil des geistigen Lebens 

bestimnt ist.

Qckes. Brockes war der erste, der sich wieder an die Natur wandte und den Versuch machte nach Massgabe der Englaender religioese und philosophiache Fragen in die Dichtung einzufuehren. Ir hatte von den Inglaendern Naturbeobachtung gelernt, die den Deut schen bisher cernfag. we es Ludwig Fulda ausdrueckt "Bisher hatte der Dichter dargestellt, was er dachte, und, wenn es hoch kam, was er fuehlte; Brockes stellte zum ersten Mal dar, was er sah. In seinen Naturbeschrelbungen geht er auf pinzelheiten ein, und er zeigt eine prosaische Natur, die zu nichts weniger als zum dichterischen Reformator geschaffen ist.

Haller ging zuerst fuer die Dichtung der zweiten schiesischen schule und der Italiener ein, aber spaeter, nachdem er durch Frankreich, Fngland und Holland gereist, wandte er sich mit Begeisterung zur ernsten englischen Dichtung. In seinen Gedichten werden die philosophischen Gedanken und Stiwemungen seines Zeitalters ausgedmeokt. Pope und Brockes namm or vielfach zum vorbilde, aber er uebertraf den ersteren an Erhabenheit der Gedanken, den letzteren an Naturbeschreibung. Bei Haller tritt das bloss Beschreibende in den Hintergmund. In seinen "Alpen" legt er mehr Gewicht auf die elegische Sehnsucht nach der Natur und Sitteneinfalt der Alpenberohner,als auf die Naturbeschreibung. Opitz hat das Lehrgedicht in die deutsche Literatur eingefuehrt, aber Haller brachte das philosophische Lehrgedicht zur Geltung. obgleich er oft der deutsche Pope genannt wird, und mit inrm den Inhalt des Lehrgedichts insgemein hat,so armte er Pope dennoch nicht nach. Wahrheit und Jeberzeugung ist in jedem seiner Verse zu erblicken. Seine gediente sind ernst und gedankenvol? und seins sprache schwerfaellig und oft unhamonisch. Aber wie schil- 

ler ueber ihn urteilt, "ueberwiegt ueberall zu sehr der Begriff in seinen Gemaelden, sowie in inm selbst der verstand ueber die Mmpriadung den Meister spielt. Daher lehrt er durchgaengig mehr als er darstellt. Fr ist gross, kuehn, feurig erhaben,zur Schoenheit hat er sich selten oder niemals erhoben." *

Hagedorn.

Hallers schwerfaelligen Dichtungen gegenueber stehen Hagedorns heitere Frzaehlungen. Fr stand auch nehr oder weniger unter dem englischen Einflusse, aber sein Hauptfreund, sein Lehrer und Begleiter war Horaz. Natuerlich in Bmprinden und Ausdruck fuehrte er die Horazische Poesie aus der schule ins Leben and wurde dadurch der vater der deutschen Anakreontik. Hagedorn uebte oinen grossen Finfluss auf die gesamite Lyrik des folgenden Zeitalters aus, denn auf seinenschultern stehen die Horazianer, die neuen Anakreontiker und die Fabeldichter.

Obgleich der englische Rinfluss in Deutschland festen Fuss fasste, obgleich eine Veraenderung in der geistigen Zustande des Volkes eintrat, die bestirnt war, die Deutschen von der Nachahmung des franzoesischen Geschmackes $z u$ befreien, lebte der franzoesische classioismus in Deutschland doch weiter,ja ex erreichte sogar aeusserlich einen Hoehepunkt in dem schriftsteller, der als Wictator in der Litteratur galt: Johann Christoph Gottsched. Loitsched als Johann Christoph Gottsched (1700-1766), Professor an der Leipziger Universitaet, stand ganz und gar unter dem Einflusseder Wolf'gchen Philosophie. Verstandesmaessigef Klarheit,wie er sie in dieser philosophie fand, wollte er auch in sprache und Literatur zur Geltung bringen. Sein hoechstes Ziel war, die Lit teratur zu verbessern und sie wieder in ihre Wuerde zu setzen; und da er die rranzoesische Litteratur so gut nach den Regeln der Vernunft wie der Natur geordnet fand, empelh $\rho^{a}$ er ihr Beispiel und 

Boileaus Regeln.

Er war wie Opitz der Ansicht, dass die Dichtkunst etwas tr. Iernbares sei,und dass ein Gedicht nur aus Klarheit des Denkens (d.h.nach Regeln denken),und correcter Form bestehe. Fast auf jedem Gebiet der Literatur fuehrte er eine Reforn ein. Seine "Grulalegung einer deutschen Sprachirunst" und "Beitraege zur kritischen Historie der deutschen Sprache ,Poesie und Beredsamkeit" haben die Kenntniss der Sprache und Literaturk gefoerdert.

'ersuch oiner titischen Dichtunst.
In seinem " Versuch einer critischen Dichtkunst" hat er seine Grundsaetze der Poesie insystematischer Form herausgegeben.

Auch in seiner Reform der Buehne und des Dramas hatte er vieles geleistet. Racine, Molierqund corneille waren seine Muster auf dem dramatischen Gebiete. In 1727 entsprach in Deutschland kein einziges Drama den drei Einheiten zeit degts undan Handlung. Gottsched machte es sich zur Aufgabe, daher, Uebertragungen von franzoesischen Dramen zu empfehlen, und er selbst verdeutschte Racines Iphigenie und trieb seine Schueler zur Uebersetzung an. pine bleibende Abhaengigkeit von der franzoesischen Dichtung herbeizufuehren, wax nicht Gottscheds ziel. Aber der bisherigen Roheit gegenueber fand die tinfuehrung des hochentwickelten franzoesischen Kunstaramas grossen Beifall in Deutschland. Gottsched verdraengte auch die oper wegen der Unnatuerlichkeit des Singens. Mit Hilfe der Schauspielerin Karoline Neuber verbannte er auch den Harlekin von der deutschen Buehne •

Gottsched durch die therde seiner Leipziger Professur verhalf dem Dichterstand zut, heeheren stufe. Er war wie opitz kein dichterisches ralent,aber er stellte doch eine hoehere stufe dar. Fr drang auf Nacharmung der Natur als die besondere Art des po- 

etischen Ausdruckes. Was Gottsched aber in den Franzosen berrunderte und nachamte, war nicht Begeisterung und Leidenschaft, son dern die Fleganz der Aussprache und. Regelmaessigkeit der/Form. Was er Nachahmung der Natur nannte, war die Verbannung von a1lem, was dern Gebraeuchlichen, dem Gewoehnlichen fern lag. Was er unter dem moralischen Giel in der Poesie verstand, var die Pflege des aesthetischen Gefuehls, des Widerwillens gegen alles Rohe, Anstoessige und Abschreckende. Gottscheds Grundsaetze und Theorien entsprechen jedoch dem herrschenden $1 i^{t}$ terarischen Geschmack des siebzehnten Jahrhunderts. Nuechterner Fomalismus, Verehrung der Worte und. Phrssen, sclavische Nachahmung, voellige Abwesenheit des maennlichen Gefuehls, der originalitaet und Inspiration sind die charakterzuege der litterarischen Producte des siebzehnten und des ersten zehntels des achtzehnten Jahrhunderts.

Zu einer Zeit,als das Volk eine grosse geistige Thaetigkeit

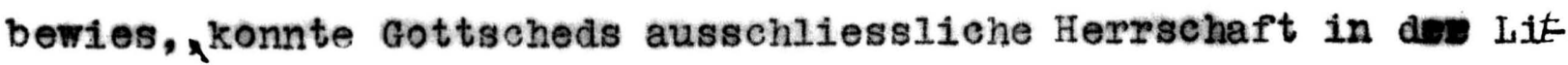
teratur nicht lange walten. Wenn Leipzig unter der Obsicht Gottscheds den Brennpunkt des franzoesischen Geschnackes bildete, so wurde Zuerich in der schweiz der Eentralpukt des englischen Einflusses. Dort fanden die englischen Schriftsteller, besonders Milton und Shakespeare grossen Beifall.

Es befinden sich also in Deutschland zwei Richtungen, die elnander feindlich gegenueberstehen,. Die eine augenscheinlich die staerkere, die dominirrende, aber in Wirklichkeit ohne allen innerlichen Gehalt, die andere bis jetzt ohne Autoritaet in der Literatur,aber voller kraft und volkstuemlichkeit. Fin Kampe zwiechen denpibelden masste entstehen, und die letztere Richtung musste siegen. 

V. KAMPF ZWISCWNN FRANZOESISCHEN

UND ENGLISCHEN EINFLUESSEN.

Die

chiveizer.

Die erste Opposition gegen Gottscheds Theorien kamen $\nabla$ on den Schweizern Bodmer und. Breitinger, die eine grosse vorliebe fuer Addison und besonders fuer Milton gefasst hatten. Vor dem Jahre 1740 lebten Gottsched, Bodmer und Breitinger in fast voelliger Eintracht. In 1723 ershien das von Bodmer und Breitinger gestiftete Blatt "Die Discurse der Mahlert", eine Wochenschrift ueber 1itherarische Gegenstaende. In 1724 uebersetzte Bodmer Mil. ton's "Paradise Lost", aber in 1739 uebersandte Breitinger dem Gottsched seine ebengeschriebene " Kritische Dichtkunst" "was den Ausbruch des Kampfos herbeibrachte. In den folgenden zwei Jahren erschienen Bodmers "Kritische Abhandlung von dem Wunderbaren in der Poesie und dessen Verbindung mit dem WahrscheinIichen" und "Kritische Betrachtung ueber die poetischen Gemaelde der nichtern.

Kritische Dichtinget.

Das Grundbuch der schweizerischen Kunstrichtung ist Breitingers "Kritische Dichtikunst." In diesem Buch betont er scharf die Gewalt der Phantasie - Der erste Band handelt ueber das Hesen der Poesie, ueber die Wahl der Umstaende, den Vergleich der Malerei und Dichtkunst, ueber das Wunderbare und wahrscheinlich in der Poesie und die Idealisimung der irdischen wesen durch de? D1chter. Der zweite Band beschaeftigt sich mit den Aeusserlichen. der poetiechen Technik,mit den worten, der Schreibart und dem Bau der verse.

Theorion der

Den Kern des Untersoniedes zwischen den Gottschedianern Schweizer. und den schweizern trifft das sechste Kapitel des Buches "von 

dem Wunderbaren und wahrscheinlishen" in der Poesie. Breitinger glaubt, dass das Wunderbare die spitze der Poesie sei. Das Wunderbare, soweit es sich in die Grenzen des wahrscheinlichen einschraenkt. "Das Wunderbare muss die Farbe der Wahrscheinlichkeit haben und die Wahrscheinlichkeit die Farbe des Wunderbaren". In der verbindung des Funderbaren mit dem whescheinlichen besteht die vornehmste Schoenheit und Kraft der Poesie. Die Poesie ist "ine Nachahmung der sehoepfung und der Natur, nicht nur in dem Wirklichen, sondern auch in dem Moegiichen. Die eigentuemliche Kunst des Poeten besteht darin, dass er die Sachen, die er dureh eine Vorstellung angenerm nachen will, von dem Ansehen der wahrhelt bis auf einen gevissen Grad kuenstlexisch entfernt, jedoch muss man den schein der Wahrheit in der allerweitesten Entrernung nicht gaenzlich verlieren.

Die Betonung der Phantasie und des Tunderbaren Puehrte zur Thnorhebung der aesopischen Fabel zur hoechsten dattung der Poesie.

Wichtig ward fuer den Versbau Breitingers Kapitel"von dem Bau und der Natur des deutschen Verses". Mr untersuchte den Alexandriner und fand, dass derselbe fuer ein langes Gedicht monoton wird. Fr hob den Gxund hervor, warum der franzoesische 8lexandriner fuer die deutsehe sprache nicht geeignet ist, und or ompeielt, den relmlosen vers, und zwar den fuenffuessigen Jambus, ohne die caesur nach der vierten silbe,mit verschiedener caesur. Breitinger konnte die Anrut des Reimes durchaus nicht finden. "Fs ist ",erklaert er, "ein alter Kirmess-Tanz, wo die Personen Uei bestirmten Pausen aus Freudebezougung in die Haende klatsohen, und man koennte den Reim fuer eine Nachamung dessen ausgeben, dadurch er aber sich alleine in einigen lustigen Gedichten einen 

Platz fordern koennte." *

Gottscheds Gottscheds Principien ueber die Poesie legte er in seinen

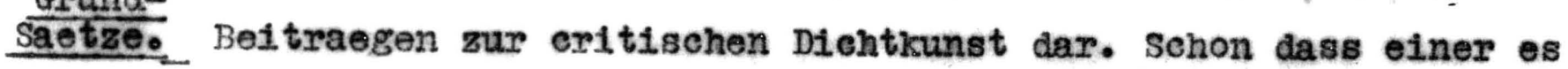
wagte, eine andere kritische Dichtkunst zu verfassen, und dabei seine ausschliessliche Autoritaet anzugreifen, war Gottsched. hoechst unangenehm.

In Bekaempfung des lohenstein'schen Sehwulstes stirmaten Gottsched und die schweizer ueberein,aber veber das wesen der poesie hatten Breitinger und Gottsched ganz verschiedene Ideen. Den prosaischen, nuechternen Wesen Gottscheds war die Pflege des Wunderbaren, des Uebernatuerlichen tief verhasst, und jede erhoehte Redeweise, die sich nicht bloss verstandesmaessig fassen 11ess, wur de als Unnatur verworfen. Ein Gedicht ohne Reim konnté sich Gottsehed schwerlich vorstellen. Und im Versmass holt er sich streng an die franzoesischen Regeln. Gottsched stand ganz entschieden unter dem Finflusse der Franzosen. Breitinger unter dem der EngIander.

Ausgang des Kampres.

Die Theorien der Schweizer waren den juengeren, strebenden Geisterm ampathisch und Gottsched sah ein, dass or keinen HaIt mehr habe. In wirklichkeit war Gottsched nicht von den Schweizern besiegt, sondern die Żeit gchritt ueber inn hinweg. Der Ausgang des Streites bezeichnet den sieg der neueren theorien, die $\nabla$ on dem englischen winfluss herruehrten,ueber die aelteren Ideen, die opitz eingeruehrt hatte. Gottsched war eine gute Vorschule gewesen. Er brachte wieder Reinigung und ordnung in die Litteratur, aber der Einfluss Brockes, Drollingers, Hallers, Hagedorns und der moralischen wochenschriften hatte den volkstuemlichen Simn doch genug gestaerkt,um die engen Fesseln des franzoesischen classieismus zu sprenge tr.

Gottsched fand noch einige Anhaenger, die unter der Leitung * Lemcke, "Geschichte der deutschen Dichtung". Seite 413. 

Leipziger Dichterverein.

von Professor Schwabe eine schrift unter dem Titel "Belustigungen des Verstandes und witzes" herausgaben. spaeter jedoch,als diese die schraeche ihres Meisters orkennen,kehren sie im den Ruecken und vereinigen sich gu dem "Leipziger Dichterverein." Sie gruendeten dann eine andere $1 i_{1}^{\frac{1}{t}}$ erarische schrift, "nie Bremer Beitraege" genannt. Die bedeutendsten Mitglieder des Bumdes waren der Satiriker Rabener,der Dramatiker J. Elias Schlegel und der populaere Moralprediger und Fabeldichter Christian Fuerchtegott Geliert.

Gellert und ate Bremer Beitraege beginnen eine neue volkstuemliche Dichtung, und es komnt nun der grosse volksaufschmung unter Friedrich dem Grossen. Was die Schweizer begonnen hatten, findet seine Vertiefung und Frfuellung in Klopstock, Wieland, Iessing und winkelmann.

Weder Bodmer noch Gottsched waren echte Poeten und konnten daher ihre Theorien nicht practisch anwenden. Die Sehweizer sahen ein , dass es nicht genuegend sei, Regeln der Dichtkunst aufzustellen, ohne durch diese entsprechende Werke zu schaffen. Bodmer dichtete daher seine "Noachide", die jedoch nicht gluecklich ausfiel. Die sehweizer erwarteten also gespannt die zolt, wann ein wahrer Dichter entstehen und ihee Theorien und Ideale in einen Werke practisch verkoerpern solite.

liopstocks Messige.
Die zeit war nicht reit entfernt; denn im Jahre 1748 erschienen in den Bremer Beltraegen die ersten Gesaenge von Klopstocks Messias, die in Inhalt, Fomn und Art den sehweizern voellig zusagten und von imnen mit enthusiastischem LOb enpfangen wurden. Bodmer war ganz entrueckt ueber das werk, und er teilt seinen Freunden mit,dass "ein Dichter lebe, auf dem Milton's Geist muhe: 

Die Messiade ist der Ausdmek des Pietiamus und vertritt die re-. ligleese stimmung der Zeit. Ihr zweek ist die verteidigung und Verherrlichung der Religion. Obgleich den Verfasser Milton als Vorbild vor den Augen schwebte, hat er die Kraft der Handlung. des "Paradise Lost" ganz verfehlt.KIopstock war vorzueglich Lyriker,imn fehlte alles als Epiker. In seinem Epos ist daher der lyrische Zug vorwiegend. In Bezug des porm des Epos waren Homer und virgil massgebend,und Klopstoek ersetzt den franzoesischen Alexandriner durch das antike Versmass, den Hexameter. Durch die Finfuehrung des antiken heroischen Versmasses, des Horazischen lyrischen Versmasses und durch die Frfindung der freien Silbenmasse, schuf KIopstock eine neue Dichtersprache.

Wenn es dem Messias auch an Handlung,an sinnlicher Begreifie

lichkeit fehlt, so war doch der Ausdruck des tỉefon religioesen Gefuehls in warmer, begeisternder Sprache eine voelifige Abweichurg von der nuechternen, kalten, verstandesmaessigen Poesie eines Opitz und Gottsched, und die Nation empfing das Gedient mit enthusiastischer Bewunderung und Teilnahme. Die begeisternde Sprache und der erhabene Ton des Gedientes gent schon aus den ersten strophen hervor, die auch zurleich den Inhalt des Messias andeuten:

"Sing', unsterbliche Seele, der suendigen Menschen Fr-

$$
\text { 10esung, }
$$

Die der Messias auf Frden in seiner Menschheit vol1endet,

Und durch die er Adams Geschlecht zu der Liebe der Gottheit,

Leidend getoedtet, und verherrilehet, wieder erhoeht hat. Also geschah des Iwigen wille. Vergebens ermub sich 

Satan gegen den goettlichen Sohn;urasonst stand Juda Gegen ihn auf:er that's, und vollbrachte die grosse

Versoelmung.

Aber, 0 That, die allein der Allbarmherzige kennet, Darf aus dunkler Ferne sich auch dir nahen die Diohtkught?

Weihe sie, Geist Schoepfer, vor den ich hier still anbete,

Fuehre sie mir , als deine Nachahmerin, voller

Entzueckung,

Voll unsterblicher Kraft,inferklaerter schoenheit entgegen.

Ruesto mit deinem Feuer die, du, der die Tiefen der Gottheit:

Schaut, und den Menschen aus Staube gemacht zum Tempel sich heiligt!

Rein sei das Herz!so dare ich, obrohl mit der bebenden Stime

Fines Sterblichen, doch den Gottversoehner besingen Und die furchtbare Bahn, mit verziehenem Straucheln, durchlaufen."

klopstock war von der tiefsten Bhprindung ergriffen, von den erhabensten Gedanken und hoechsten Idealen erfuellt und von einer Liebe zur Religion, Natur und zum Vaterland begeistert. Fr schwebt aber die Haelfte der Zeit in den wolken und besingt die Freuden der Unsterblichkeit. Seine Gedichte sind daher dem Leser von hemtzutage oft langweilig und eintoenig. Klopstock begann jedoch mit selnem Messias eine neue Epoche in der Literatur, und dass er bei dem volke solchen Beifall fand, deutet auf 

die sentirentale Geistesstirmung der Zeit hin. Das volk suchte geistige Nahmung und fand sie in der Messias.

Die Fesseln des

PseudoCIssic1s-

mus gesprengt.

Als Klopstock wieder Interesse fuer Deutschlands nationale

Vergangenheib erweckte,als er gemanische Helden dem volke wieder vorfuehrte,und den Gebrauch der roemischen und griechischen Goetternamen durch den derl germanischen zu ersetzen suchte, war die Jitieratur von den ongen Fesseln des Pseudo- Classieismus befreit, und der erste schritt zur wiederherstellung einer Nationa.1.itteratur gethan. Milton, Shakespeare, voltaire, Rousseau und Diderot ueben immer noch, einen grossen Einfluss auf die I itheratur aus, aber man findet von nun an keine sklavische Nachamma

Gruende fuer klopstocks Errolg.
Priedrien der Grosse.

der englischen und franzoesischen vorbilder.

Wenn man den grossartigen Beifall und den Binfluss K1opstocks bedenkt, stellen sich die Fragen heraus:

1). Wamun wurde er von dem volke so begeistert aufgenommen?

2). Was set ate Klopstock in den Stand, eine neue Ipoche einzufuehren?

dieser Fragen

Die Beantwortung ${ }_{\Lambda}$ findet man in der Betrachtung folgender

\section{Gruende:}

a). Der Finfluss Friedrich des Grossen auf Deutsehland.

b). Der geistige Zustand des Volkes.

c). Fngland und Frankreich als vorbereitende Einfluesse.

Das Heranwachsen Preussens zu einem einflussreichen staat Europas, die Thaetigkeit, Selbststaendigkeit und der Finfluss dizses kleinen Reichs, sowie der merkwuerdige politische und militaerische Erfolg Friedrich des zweiten, seine liebe zur Freiheit und sein Interesse an Philosophie und Iiteratur im allgemeinen, emweckte in dem volke einen Patriotismus und oinen volkatuem- 

lichen stolz, don Deutschland seit Jahrhunderten nicht gespuert hatte. Friedrich der Grosse verachtete zwar die deutsche Iitetur,aber er war ihr von grossen Diensten, indem er die Gmundiage zur nationalen Finheit legte,aus welcher allein eine volkstuemiiche itteratur entstehen konnte.

Das geistige Der geistige Zustand des Volkes wurde durch den Rinfluss des Leben.

Pietismus und Rationalismus, durch die englischen und franzenesischen Philosophen orveitert, und der moralische Zuatand verbessert. Die Abhaengigkeit von der Iranzoesischen Litteratur hatte doch die gute Folge, den in Fngland und Frankreich ausgebrochenen Kampe fuer Freiheit des Denkens und kritischen forschens in Deutschland fortzusetzen. Ohne den kuenstlerischen Einfluss Frankreichs waehrend Gottscheds Herrschaft, haette Klopstock seine herrlichen Leistungen nicht vollgiehen koennen. Klopstock erschien gerade zur rechten Zeit. Fr erntete,was Opitz und Gottsched gesaet hatten und wiet das volk auf eine nationale Dich tung hin.

Fs entstand bald ein anderer Dichter, ein Zeitgenosse wanKlopstock, der zugleich dessen Gegenteil und Brgaenzung bildet, Martin Wieland. Waehrend Klopstock, das Product des Pietismus, in den wolken schwebt, und die erhabenen Freuden der Fwigkeit in wamer aber schwerfaelliger sprache besingt,so wandelt wioland der dem Rationalismus zureneigt sst, unten auf der Brde herum, und drueckt sich in leichter, eleganter, anmutiger und fliessender sprache aus, ueber das Sinnliche, ueber die Freuden und Leiden des taeglichen Lebens. Klopstock erweckt Interesse unter den Buergerndud Gelehrten; Wieland in dre Gesellschaft. Er ist der popularisirende Dichter. 

Zu Klopstock und Wieland stellt sich noch ein dritter als Vorbereiter einer classischen Periode, Gottfriod Herder. Herders Ansichten ueber Philosophie, Hrziehung, Geschichte und Poesie waren von grosser Bedeutung fuer das deutsche Volk. Br erweckte den historischen Sinn, er oefmete die Bahn auf dem Gebiete der Evolution der Geschichte, und erregte wieder durch seine samIung von Volksiledern Interesse fuer diese Gattung der Poesie. Fr ging fuer Volkstuemlichkeit, Individualitaet und originalitaet ein, und sieht in dem Volk, und nicht in dem Dichter, den Ursprung aller wahren Poesie.

Nachdem diese drei Maenner erschienen waren, nachdem der moralische und religioese Ton der Dichtkunst durch Klopstock erhoeht worden, die Sprache durch Wieland eine Bleganz, Leichtigkeit und Grazie gewonnent, die sie neben die franzoesische stelzte, nachdem Herder die philosophischen Anschaurangen im Leben und in der Literatur erweitert und verbessert hatte, komnte Lessing das erste elassische Werk verfassen. Klopstock ahnte die neue Kunst;Lessing hat sie mit kritisehem Blick erfasst;Herder hat sie nit allen anderen Phasen der cultur unter eine Aussicht gebracht.

Lessing ist nun der erste wirklich classische schriftsteller. Ir kaempte heftig gegen Nachahmung der Franzosen und behauptete, dass, wenn wem die Deutschen nachahnen muessten, so solIten sie wenigstens eine Nation zum Vorbilde nemen, deren charakter wit dern der Deutschen naeher vervandt sei, naemlich die englische. Lessing begruendete eine gediegene deutsche Prosalur fuehm te eine Reform auf fast jeden Gebiete der Iitteratur ein. AIIe 

die Bedingungen zur Gruendung einer classischen Literatur, naonlich die vollendete Form der nationalen Sprache; der ausschliessIich volkstuemitche charakter der Iitteratur, und die vollendung nes Werkes zur hoechsten stufe der folwiehling auf dem 11titerarischen Gebiete,zu velcher os gehoert; alle diese Bedingungen verden zuerst in Lessing vereinigt. Der Pseudo-classicianus ist num durch den classicimus ersetzt worden. Aus den schonprwaennten Verhaeltnissen Deutschlands zur Zeit des dreissigjaehrigen Krieges kann man den Gmund der Fxistenz eines Pseudo-classicismus in der Iiteratur erkennen.

lianger an Von dem dreissigjaehrigen Kriege bis zu KIopstock und Lesifgeitundsing war Deutschland von Grund aus so erschoepft, dass von selbstDriginailtaet. staendigkeit weder in der Iiltberatur noch in der Politik die Rede sein konnte. Is entstand in dieser Zeitperiode kein Dichter, der die eigentlichen Maengel der Litteratur erkannte. Aber viele $1 i^{t}$ terarische Geister erkannten wohlyden Mangel ax einer deutschen Iiteratur, und da die Nation onne alle volkstuemilchkeit und schoepferische Krait war, und daher keine volkstuemliche Dichtung schaffen konnte, mussten die Dichter nach dem ersten besten, und vielleicht dem einzigen Mittel greifen, neemlich der Nachahmung der Li,teraturen anderer voelker und der Classiker. fa, Nacharmung! Aber wohin nur kann Nachahmung fuehren, wenn der Nachahmer nicht im stande sst, den echten wert des erwaehlten Gegenstandes zu erblicken and zu erfassen, wenn er nur die Aussenseitepie vollendung der Form, das Erkuenstelte, ins Auge fasst? Wohin sonst, als zur Hervorbringung eines falschen Productes,eines eitlen, erkuenstelten Werkes ohne allen innerlichen Wert? 

Hachahmung das inzige RettungsintteI der itteratur.

So geschah es in der deutschen Litteratur zu dieser Zeit. Nachahmung war das einzige vorhandene Mittel, nachdem die deutsche Sprache wieder ins leben gerufen war, um die deutsche titteratur zupetten. Und sie hatte die Betonung der Form und die Verachtung des innerlichen, poetischen Geistes zur Folge.

Aber es war grade durch diese Nachahnung, dass die Deutschen Selbststaendigkeit erlangt haben. Die Auslaenderei war Deutschlands tiefstes Verderben, aberfugleich der Grund seiner Rettung. Nachahrung der Form der Gedichte brachte ordnung und system in die deutsche hitteratur, was die Grundlage aller Reform bildet. Das deutsche volk ammte nicht nur die Li teratur anderer Nationen nach, sondern auch das Leben und die Denkart derselben. Auf diese Weise warde Deutschland mit den Philosophen Englands und Frankreichs bekannt, und man erwachte bald zur tinsicht, dass das Wesen der Kunst und die Volkstuemlichkeit êñander nicht ausschliessen, sondern zu einanderfgehoeren und wechselweise auf einander wirken. Die Nachammung diente der litteratur als eine stuetze, auf welche die Deutschen sich soliange verlassen mussten, bis deren Selbststaendigkeit und volkstuemlichkeit wieder hergestelit waren. Und wenn sie auch in Pseudo-classicismus ausartete,so war sie dennoch von grosser Bedeutung fuer die deutsche Lithoratur.

Waehrend der Herrschaft des Pseudo-classicismus, die sich etwa ueber einhundert Jahre hinaus erstreckte, hat die deutsche Sprache die lateinische als die Dichtersprache erstetzt; die Literatur ist gerettet worden und das beste auf diesem Gebiete geleistet-werden-, was ein gedruecktes, erschoepftes Volk leisten konnte. Die Geschichte Deutschlands vom Ende des Krieges bis gu Friedrich dem Grossen ist nichts weiter,als die der bestaen- 

digen Intwickelung eines gedrueckten aber aufstrebenden Volkes zur Selbststaendigkeit und vollendeten Bildung. Die Mittel zu dieser Entwickelung fand es in den Anregungen und Finwirkungen des freieren und vorangeschrittenen Auslandes.

Zusammen-

hang

zuischen

classischer und pseudoclassischer Periode.

Der zusarmenhang zwischen dieser und der naechsten olassischen Periode ist darin zu Pinden, dass die erstere eine wichtige vorschule fuer die letztere war. Ohne die Leistungen eines Opitz und eines Gottsched, die ordnung und System in die Literatur einfuehrten;ohne die Entwickelung des geistigen und moralischen Zustandes des volkes, welche durch die moralischen und philosophischen Anschauungen des Auslandes angeregt, und durch Leibnitz, wolf und Herder vollzogen ward ; ohne den Kampe zwischen den Schweitern und Gottschedianern, dessen Entscheidung den Sieg des nach kuenstlerischem Ausdruck sich sehnenden Volksgemuets ueber die entartete Renaissance zur Folge hatte; ohne die tinfluesse eines Klopstock, der die Theorien der Schweizer in seinen Gedichten verkoerperte, und eines wieland, der der Sprache Grazie und Aninut verlieh; ohne alle diese vorbereitenden Anfaenge waere eine classische Li teratur im achtzehnten Jahrhundert unmoeglich gewesen. Aus dem Pseudo-classicismus entwickelte sieh also langsam, aber bestaendig, der classicismus, der sich zunaechst in Lessing verkuendet und seine schoenste Bluete in schiller und Goethe erreicht hat.

St. Louis, Mo.

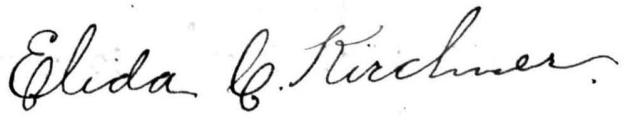

JuIy 31,3.901.

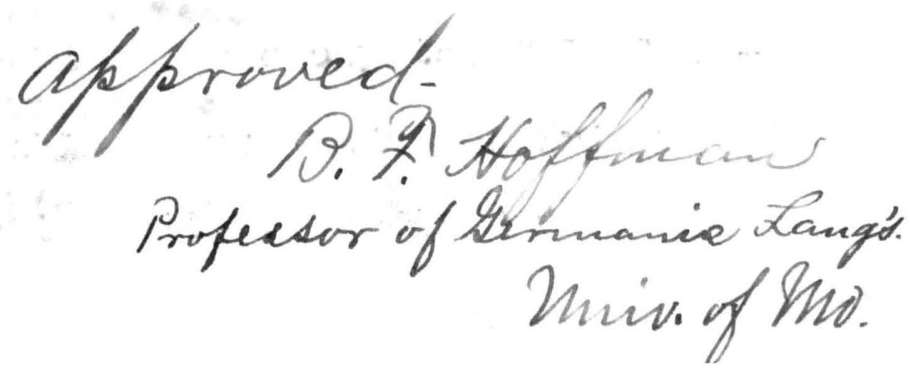





\section{BIBLI OGRAPHIE.}

Allgemeine Biographie. Seifegig. 1887

B1bllothek der Deutschen Klassiker.Hildburghausen. 1863.

Bledermann,K. Deutschland im 18. Jahrhundert. Leipzig. 1854/80. Boringki, K. Die Poetik der Renaissance und die Anfaenge der 1itterarisehen Kritik in Deutsohland. Berlin.1886. Bryoe, James. The Holy Roman mire. Sondori. 1892 Burokhardt.J. Ronaissance.in dtaly. Sondon 1878 . manoke. Kuno. soeial Farcess in German Literature. Kew Yoak. 1896. Frertagequstar. Dodtor Iuther. Boston. 1894

Gervinus,G. Geschionte der deutschen Dichtung. Seipzig. 1871.

exnoe, 0.F. Leben und Prerke deutscher Dichter. Seipgig. 1872. Ham, Terner. Geschichte der poetischer Sitteratur der Dentschen. Berlin. 1890. [Banschmeig. 1894. Hettner,H. Geschichte der deutschen Literatur im 18.Jahrhundert Klugen.H. Geschichte der deutschen National-L1,teratur. alteubug. 1876.

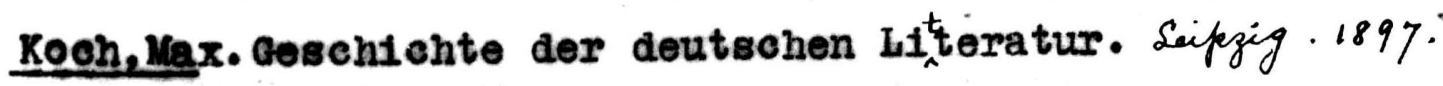
Koentg, R. Deutsche Litteraturgeschtchte. Bielefeld und Leipzig.

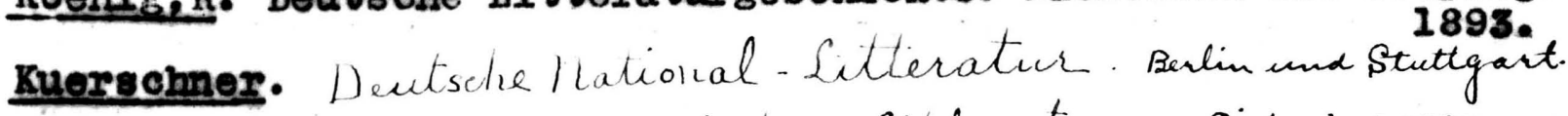
Nurze H. Geschichte der dentschen Siltiratur. Seipzig. 1887. Lemeke,k. Von Op1tz bis Klopstook. Leipzig.1882. Herinson,Henry. A. Sketeh of Herder and his Times. London.1884. Perry, Pseudo-classioism, in lsermany.

Sehore, Joh. Allgemeine Geschichte der literatur. Stuttgart 1869. Sobnidt, J. Geschichte des geistigen Lebens in Deutschland von Letbittz bls aue Lessings Tod. Supzig. 1862.

vilmared. Te ceschichte der deutschen rational-Iitieratur. varburg und Le1pzig. 1868. 



RECEIVED NOV 291909 UNrv of Mo.

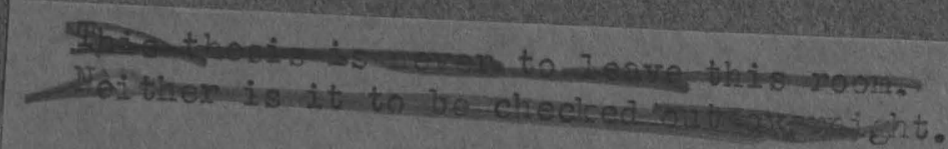


